\title{
Characterization of the Line Configuration in Wired Communication Networks
}

\author{
Kazi Moinul Islam
}

\author{
A Thesis \\ in \\ The Department \\ of \\ Electrical and Computer Engineering
}

\author{
Presented in Partial Fulfillment of the Requirements \\ for the Degree of Master of Applied Science at \\ Concordia University \\ Montréal, Québec, Canada
}

October 2011

(C) Kazi Moinul Islam, 2011 


\section{CONCORDIA UNIVERSITY}

School of Graduate Studies

This is to certify that the thesis prepared

By: $\quad$ Kazi Moinul Islam

Entitled: Characterization of the Line Configuration in Wired Communication Networks

and submitted in partial fulfilment of the requirements for the degree of

\section{Master of Applied Science}

complies with the regulations of this University and meets the accepted standards with respect to originality and quality.

Signed by the final examining committee:

Dr. Rabin Raut, Chair

Dr. Youmin Zhang (MIE), External

Dr. Wei-Ping Zhu, Examiner

Dr. Amir G. Aghdam, Supervisor

Approved by

Dr. W. E. Lynch, Chair of the ECE Department

2011

Dr. Robin Drew, Dean of Engineering and Computer Science 


\begin{abstract}
Characterization of the Line Configuration in Wired Communication Networks
\end{abstract}

Kazi Moinul Islam

This thesis presents an algorithm to identify the full configuration of a wired transmission line from its frequency response. It is assumed that the line can have up to two bridged taps. Each bridged tap divides the main line to two segments, and with two bridged taps there will be at most three segments in the main line. Furthermore, each segment of the main line and the bridged taps can have three different gauges. The problem of characterizing the line configuration is concerned with identifying each segment (main line segments and bridged taps) in terms of its length and gauge. The problem is solved in two phases: initialization and optimization. The algorithm can be used as single ended line testing, which means the line can be characterized by performing a simple test from the central office. Simulations demonstrate the accuracy of the proposed method. 
I dedicate this work to my father, Kazi Nazrul Islam, because of his exceptional support and encouragement for my study from my childhood. 


\section{Acknowledgements}

Thanks are due first to my supervisor, Dr. Amir G. Aghdam. Because he gave me the opportunity to work for this interesting project. Moreover, I am really greatful to him for his close supervision during my work to solve this complex problem. I also got lots of help from his past work that was done by him to characterize the line configuation with single bridged tap. I would also like to thank to my colleagues Farzad Salehisadaghiani and Mohammad Salehizadeh for their valuable suggestions time to time, during my work. 


\section{Table of Contents}

List of Figures . . . . . . . . . . . . . . . . . viii

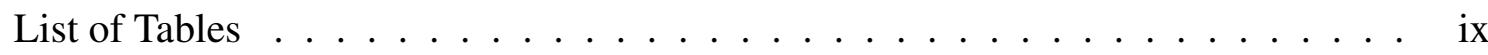

List of Abbreviations ....................

1 Introduction 1

1.1 Background .......................... 1

1.2 Frequency Response of a Single Transmission Line . . . . . . . . . . . . 3

1.3 Two-Port Characterization of Transmission Lines . . . . . . . . . . . . 5

1.4 Two-Port Characterization of Bridged Taps . . . . . . . . . . . 8

1.5 Frequency Response of a Subscriber Loop with Multiple Segments . . . . 9

1.6 Frequency-Dependency of the RLCG Parameters . . . . . . . . . . . . . 11

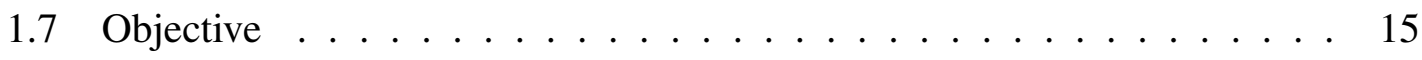

1.8 Contribution and Publication . . . . . . . . . . . . . 16

2 Determination of the Lengths of the Bridged Taps and the Total Length of $\begin{array}{ll}\text { the Line } & 17\end{array}$

2.1 Effects of Different Transmission Line Segments _ . . . . . . . . . . . 17

2.2 Determine the Lengths of the Bridged Taps _ . . . . . . . . . . . . . 19

2.3 A Method to Determine the Total Length of the Transmission Line . . . . 22

2.3.1 Simulation Result for Initial Length Estimate of the Main Line . . 23

2.4 Frequency-Dependency of the $R L C G$ Parameters . . . . . . . . . . . 25

$3 \quad$ Identification of Line Configuration $\quad 30$

3.1 Finding an Initial Estimate for Line Configuration . . . . . . . . . . . 30 
3.2 Search for the Optimum Configuration . . . . . . . . . . . . . . 32

3.3 Reducing Program Execution Time . . . . . . . . . . . . . 35

3.4 MATLAB Programs . . . . . . . . . . . . . . . . 36

3.4.1 Steps or Pseudocode of the Initialization Phase . . . . . . . . 36

3.4.2 Steps or Pseudocode of the Optimization Phase . . . . . . . . 37

3.5 Simulation Results . . . . . . . . . . . . . . . . . . 39

4 Conclusions $\quad 47$

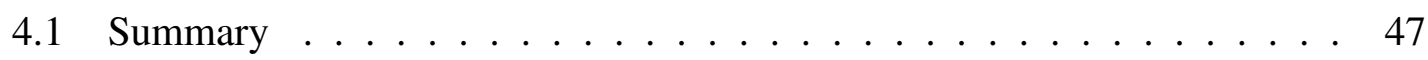

4.2 Future Work . . . . . . . . . . . . . . . . . . 48

Bibliography $\quad \mathbf{5 0}$

A Appendix $\quad \mathbf{5 6}$

A.1 MATLAB Code to Determine the Length of Bridged Taps . . . . . . . . . 56

A.2 MATLAB Code to Determine the Total Length of Line . . . . . . . . . 58

A.3 MATLAB Code to Deal with the Variable Nature of $R L C G$ Values with Frequency ........................ 60

$\begin{array}{lll}\text { B Appendix } & 63\end{array}$

B.1 Modified Section of MATLAB Code for the Initialization Phase . . . . . 63 


\section{List of Figures}

1.1 A two-port network model of a twisted pair transmission line. . . . . . . 4

1.2 Incremental section of the twisted-pair transmission line. . . . . . . . 6

1.3 An example of a subscriber loop from the central office to the customer premise equipment, with only one bridged tap connected to the main line. 9

1.4 Subscriber loop with two bridged taps. . . . . . . . . . . . . . . . 10

1.5 Resistance vs frequency for a 26 gauge cable. . . . . . . . . . . . . . 12

1.6 Resistance at low frequencies for a 26 gauge cable. . . . . . . . . . 13

1.7 Inductance vs frequency for a 26 gauge cable. . . . . . . . . . . . . . . 14

1.8 Inductance at low frequencies for a 26 gauge cable. . . . . . . . . . . 14

2.1 The effect of a bridged tap in the transmission line. . . . . . . . . . 18

2.2 The magnitude response of a loop with single bridged tap. . . . . . . . 20

2.3 The magnitude response of a loop with two bridged taps. . . . . . . . . . 21

2.4 The real magnitude response and the associated fitting polynomial of order 10 for Configuration $1 . \ldots \ldots . \ldots . \ldots 24$

2.5 The real magnitude response and the associated fitting polynomial of order 10 for Configuration 2. . . . . . . . . . . . . . . . 25

3.1 The flowchart of the proposed algorithm. . . . . . . . . . . . . 34

3.2 Magnitude response for the transmission line of Example 1. . . . . . . . 39

3.3 Magnitude response for the transmission line of Example 2. . . . . . . . . 41

3.4 Magnitude response for the transmission line of Example 3. . . . . . . . . 43

3.5 Magnitude response for the transmission line of Example 4. . . . . . . . . 45 


\section{List of Tables}

1.1 Parameter values for a 26 gauge twisted pair cable . . . . . . . . . . 15

1.2 Parameter values for a 24 gauge twisted pair cable . . . . . . . . . . . 15

$2.1 R L G C$ values for a 26 gauge twisted pair cable . . . . . . . . . . . . . 27

$2.2 R L G C$ values for a 24 gauge twisted pair cable . . . . . . . . . . . . 28

$2.3 R L G C$ values for a 22 gauge twisted pair cable . . . . . . . . . . . . . 29 


\section{List of Abbreviations}

DSL Digital Subscriber Line

VDSL Very high speed Digital Subscriber Line

POTS Plain Old Telephone Service

QoS Quality of Service

CO Certral Office

CPE Customer Premise Equipment

ONU Optical Network Unit 


\section{Chapter 1}

\section{Introduction}

\subsection{Background}

Telephone lines were originally installed for voice traffic. Since their original deployment (which goes back as far as 1881) there has been a technological revolution in the communications industry. In particular, development of digital subscriber line (DSL) technology in 1989 has opened up a new dimension in wired communication, enabling the transmission of megabits of data per second over the same twisted pair channel which is used for plain old telephone service (POTS) [1], [2], [3], [4]. The operators are now generating revenues from voice service (at low frequency) and high speed data service (at high frequency) using the same infra structure. 
DSL services can be classified as symmetric and asymmetric [1]. In a symmetric DSL service, transmission of data in both downstream and upstream directions is performed in the same frequency band. In an asymmetric DSL service, on the other hand, a higher transmission rate is assigned to the downstream channel, and there is no overlap between the downstream and upstream frequency bands [1]. Each service type has its own advantages and disadvantages in terms of quality of service (QoS), robustness, etc. whose details are not in the scope of this report. However, they both suffer from a number of issues related to the physical characteristics of the channel. For example, some of the problems reported by the DSL customers are directly or indirectly related to the line configuration, and in particular to the characteristics (location and length) of any bridged tap which may exist in the line [4]. Bridged taps are open-circuited twisted pairs which are used by the telephone companies to increase the flexibility in providing service to customers [1]. However, they can potentially degrade the frequency response of the channel, specially in high frequencies (which is normally used for DSL services) [4], [5]. If the bridged tap is very long (e.g., the same length as the main line), it introduces approximately a flat attenuation of $3.5 \mathrm{~dB}$ in the loops frequency response [48].

Many of the DSL service problems are related to the line configuration which was implemented before DSL was invented. It is not feasible to make fundamental changes in the telephone line network configuration in order to obtain a more suitable network for DSL service. It is also not cost-efficient to hire technicians to run tests and find the source of the problem after each trouble report, if it can be identified simply performing a test from the central office $(\mathrm{CO})$ [6], [7]. Characterization of the transmission line is important for network monitoring and maintenance. It can also help increase the number 
of customers that can be provisioned for DSL, or increase the average bit rate of existing customers. Some of the new DSL services require higher bandwidths, bit rates, and reliability, which can only be assured by having accurate knowledge of the lines [18].

In this work, it is desired to develop a program which can be used to fully characterize a transmission line, using some information (about the frequency response of the line) which can be collected at the CO. The full characterization means determining the number of different line segments as well as the length and gauge of every one of them. Each segment can have three different gauges, and each bridged tap segment has prescribed upper and lower limits for its length. It is also assumed that each transmission line can have at most two bridged taps (this assumption is in accordance with the DSL standard loops). The proposed characterization algorithm performs in two phases: initialization and optimization.

\subsection{Frequency Response of a Single Transmission Line}

Twisted-pair transmission lines can be modeled as a two-port (or $A B C D$ ) network as shown in Figure 1.1 [1]. The voltage and current of each port depends on the source and load impedances satisfying the following matrix relation:

$$
\left[\begin{array}{l}
V_{1} \\
I_{1}
\end{array}\right]=\left[\begin{array}{ll}
A & B \\
C & D
\end{array}\right] \cdot\left[\begin{array}{l}
V_{2} \\
I_{2}
\end{array}\right]=\Phi \cdot\left[\begin{array}{l}
V_{2} \\
I_{2}
\end{array}\right]
$$




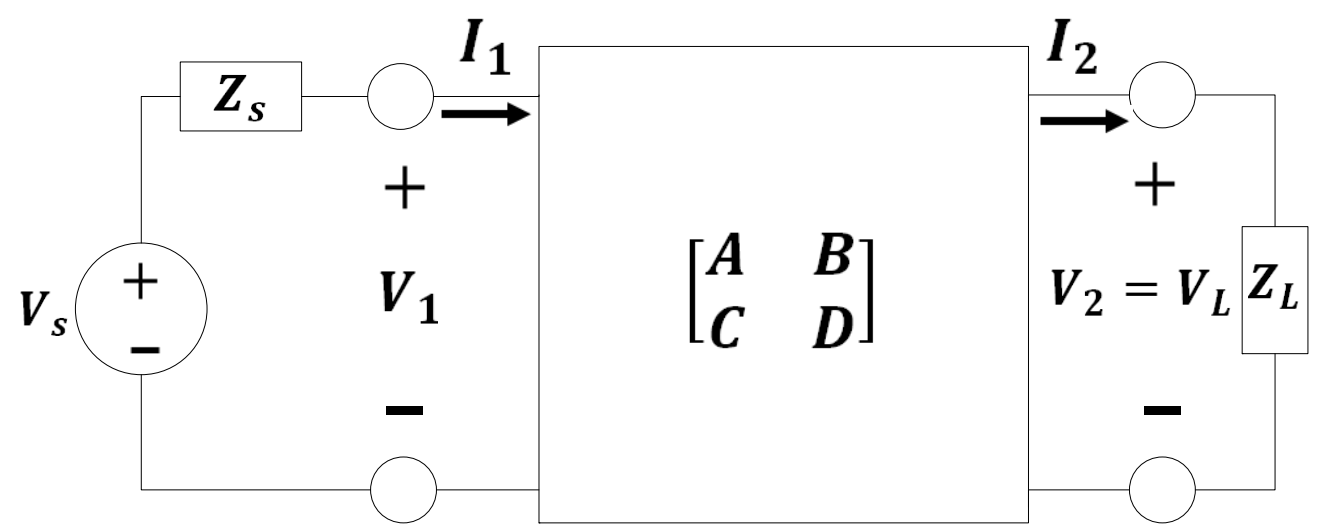

Figure 1.1: A two-port network model of a twisted pair transmission line.

where $\Phi$ is a $2 \times 2$ matrix of the four frequency-dependent parameters $A, B, C$ and $D$. All of these parameters depend only on the network and not on external connections, and are given by:

$$
A=\left.\frac{V_{1}}{V_{2}}\right|_{I_{2}=0} ; B=\left.\frac{V_{1}}{I_{2}}\right|_{V_{2}=0} ; C=\left.\frac{I_{1}}{V_{2}}\right|_{I_{2}=0} ; D=\left.\frac{I_{1}}{I_{2}}\right|_{V_{2}=0}
$$

The parameters $A, B, C$ and $D$ are known as open-load voltage ratio, shorted-load impedance, open-load admittance and shorted-load current ratio, respectively.

Now, consider the following function which depends on the load impedance connected at the right side of Figure 1.1.

$$
T(f)=\frac{V_{2}}{V_{1}}=\frac{V_{2}}{A \cdot V_{2}+B \cdot I_{2}}=\frac{1}{A+B \cdot \frac{I_{2}}{V_{2}}}
$$

The load impedance is defined as follow:

$$
Z_{L}=\frac{V_{2}}{I_{2}}
$$


Substituting the load impedance in equation (1.3) yields:

$$
T(f)=\frac{1}{A+\frac{B}{Z_{L}}}=\frac{Z_{L}}{A \cdot Z_{L}+B}
$$

Assume now that $V_{S}$ is the input voltage supply with finite internal impedance $Z_{S}$ as shown in Figure 1.1, and $V_{L}$ is the output voltage across the load $Z_{L}$. So, we can relate $T(f)$ to the transfer function $H(f)$ between input and output as given below:

$$
\frac{V_{L}(f)}{V_{S}(f)}=H(f)=\frac{V_{L}(f)}{V_{1}(f)} \cdot \frac{V_{1}(f)}{V_{S}(f)}=\frac{Z_{1}}{Z_{1}+Z_{S}} \cdot T(f)
$$

where $Z_{1}$ is the input impedance of the terminated two-port. The frequency response, $H(f)$ generally depends on the load and source impedances. The input impedance of the two-port is calculated by using the following equation:

$$
Z_{1}=\frac{V_{1}}{I_{1}}=\frac{A+\frac{B}{Z_{L}}}{C+\frac{D}{Z_{L}}}=\frac{A \cdot Z_{L}+B}{A \cdot Z_{L}+B}
$$

\subsection{Two-Port Characterization of Transmission Lines}

The two-port model of a twisted-pair line can be derived from the per-unit length two-port model as shown in Figure 1.2. The $R, L, C$, and $G$ parameters indicate resistance, inductance, capacitance and conductance per unit length of the transmission line, respectively. Now, let a segment of the transmission line be regarded as the cascade of such sections that are infinitesimally small in length. At any point $y$, the two-port voltages and currents are related through the following differential equations at any given frequency $\omega=2 \pi f$ 


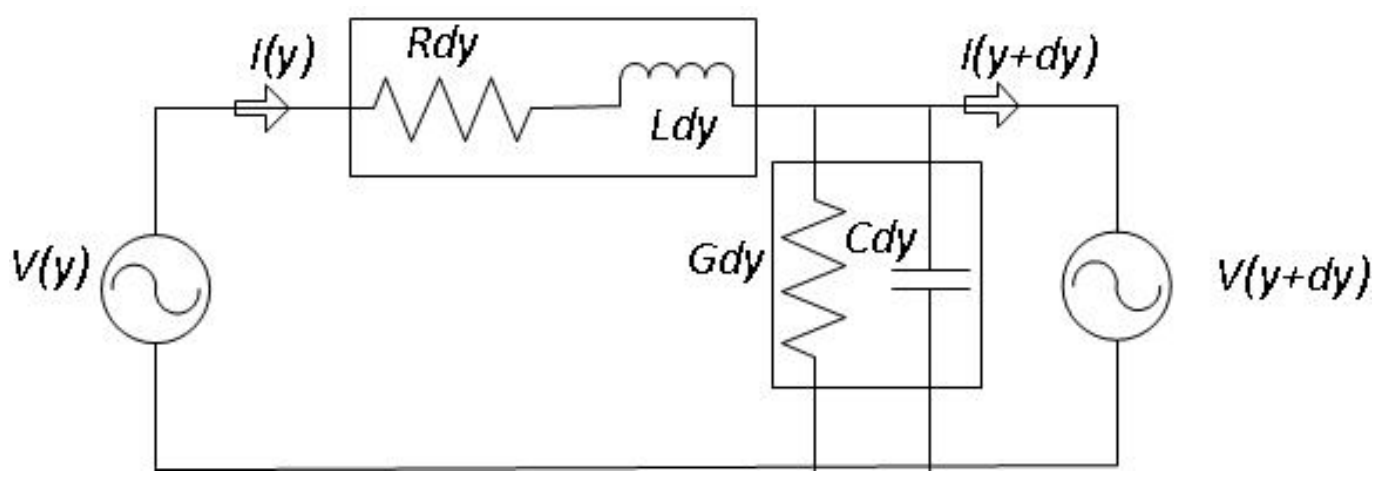

Figure 1.2: Incremental section of the twisted-pair transmission line.

[1]:

$$
\begin{array}{r}
-\frac{d V}{d y}=(R+j \omega L) \cdot I \\
-\frac{d I}{d y}=(G+j \omega C) . V
\end{array}
$$

where $V$ and $I$ are phasor quantities indicating peak amplitudes of sinusoids at frequency $f$ (the $R, L, C, G$ parameters also vary with frequency). The above equations are also equivalent to the following second-order differential equations:

$$
\begin{gathered}
-\frac{d^{2} V}{d y^{2}}=\gamma \cdot V \\
-\frac{d^{2} I}{d y^{2}}=\gamma \cdot I
\end{gathered}
$$

In particular:

$$
\gamma=\alpha+j \beta=\sqrt{(R+j \omega L)(G+j \omega C)}
$$

where $\gamma$ is the frequency-dependent propagation constant for the twisted-pair, which is used to characterize each segment of the transmission line. The solutions to the differential equations (1.10) and (1.11) are the sum of positive and negative-direction waves that vary with position as $e^{+\gamma y}$ and $e^{-\gamma y}$, where the sign of the exponent depends on direction. 
The real part $\alpha$ and the imaginary part $\beta$ in equation (1.12) are called the attenuation constant and the phase constant, respectively. For a lossless line $(R=0, G=\infty)$, the attenuation constant is zero. The solution to the differential equations (1.10) and (1.11) are as follow [1]:

$$
\begin{gathered}
V(y)=V_{0}^{+} \cdot e^{-\gamma y}+V_{0}^{-} \cdot e^{+\gamma y} \\
I(y)=I_{0}^{+} \cdot e^{-\gamma y}+I_{0}^{-} \cdot e^{+\gamma y}
\end{gathered}
$$

By substituting either of these solutions into (1.8) and (1.9), the ratio of the positivegoing voltage to the positive-going current, and the ratio of the negative-going voltage to the negative-going current are obtained, which provide a constat characteristic impedance of the transmission line. Suppose that a transmission line of length $d$ has the solution $V_{L}=V(d)$ and $I_{L}=I(d)$, which leads to the following equations:

$$
\begin{gathered}
V_{L}=V(d)=V_{0}^{+} \cdot e^{-\gamma d}+V_{0}^{-} \cdot e^{+\gamma d} \\
I_{L}=I(d)=I_{0}^{+} \cdot e^{-\gamma d}+I_{0}^{-} \cdot e^{+\gamma d}
\end{gathered}
$$

As the two voltage waves in each direction are related to the same direction current waves by the characteristic impedance $Z_{0}$, one can solve the above two equations for $V_{0}^{+}$and $V_{0}^{-}$ to arrive at:

$$
\begin{gathered}
V_{0}^{+}=\frac{1}{2}\left(V_{L}+I_{L} \cdot Z_{0}\right) \cdot e^{\gamma d} \\
V_{0}^{-}=\frac{1}{2}\left(V_{L}-I_{L} \cdot Z_{0}\right) \cdot e^{-\gamma d}
\end{gathered}
$$

Now, putting these constants into the solutions of (1.15) and (1.16) and evaluating voltage and current at $y=0$ in terms of those at $y=d$, the following $A B C D$ representation for the 
transmission line is obtained [1]:

$$
\left[\begin{array}{c}
V(0) \\
I(0)
\end{array}\right]=\left[\begin{array}{cc}
\cosh \gamma d & Z_{0} \sinh \gamma d \\
\frac{1}{Z_{0}} \sinh \gamma d & \cosh \gamma d
\end{array}\right] \cdot\left[\begin{array}{c}
V(d) \\
I(d)
\end{array}\right]
$$

where $\gamma$ and $Z_{0}$ are the propagation constant and the characteristic impedance of the line, respectively.

\subsection{Two-Port Characterization of Bridged Taps}

A bridged tap is an open-circuited twisted pair providing flexibility for future additions and changes in service demands, and can be viewed as a three-port network. However, one of the ports appears as a load impedance to the line between the two segments on each side of the bridged tap. Thus, a bridged tap can be described by the following two port characterization model [1]:

$$
\left[\begin{array}{cc}
1 & 0 \\
\frac{1}{Z_{b t}} & 1
\end{array}\right]
$$

where $Z_{b t}$ denotes the characteristic impedance of the line for bridged tap section. 


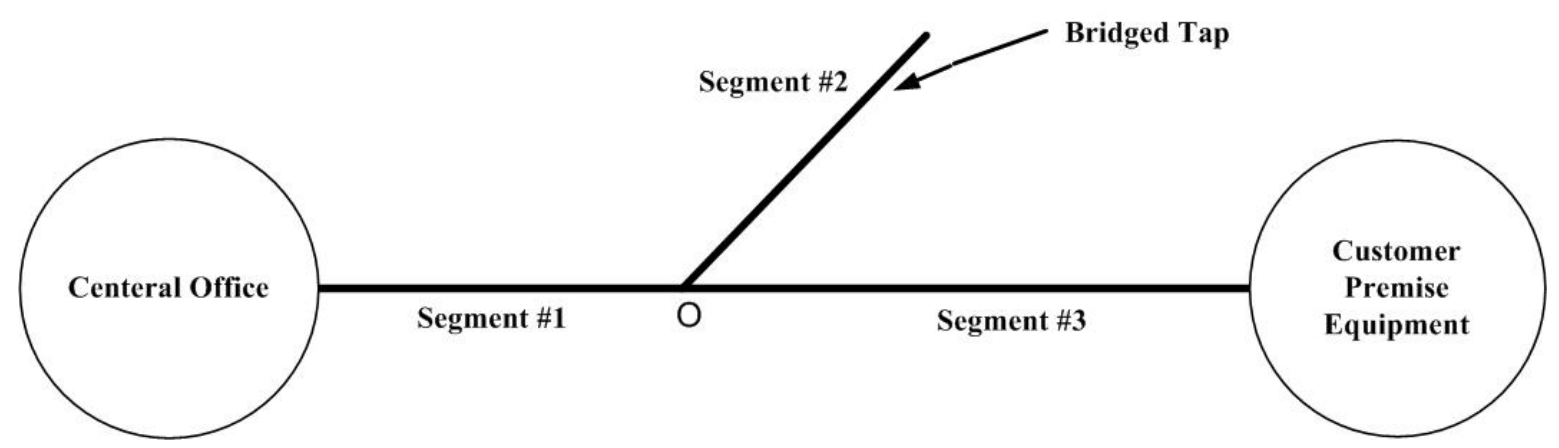

Figure 1.3: An example of a subscriber loop from the central office to the customer premise equipment, with only one bridged tap connected to the main line.

\subsection{Frequency Response of a Subscriber Loop with Mul-}

\section{tiple Segments}

It is shown in this section how to derive the frequency response of a subscriber loop with multiple segments. Consider first a transmission line with one bridged tap, which consists of three segments as shown in Figure 1.3: segment \#1 between the $\mathrm{CO}$ and the point $\mathrm{O}$ where the bridged tap is connected to the main line; segment \#2 which is the bridged tap, and segment \#3 between the connection point $\mathrm{O}$ and the customer premise equipment (CPE). The main line or main channel connecting the $\mathrm{CO}$ to the $\mathrm{CPE}$ in this case consists of segments \#1 and \#3, and is the primary contributor to the attenuation of the signal passing through the line (both uplink and downlink). The effect of the bridged tap, as discussed later, is the reflection of the signal passing through the main channel from the connection point. Figure 1.4 depicts the line configuration for the case of two bridged taps, and the five segments associated with it. The problem of the characterization of the line configuration is concerned with identifying the length and gauge of each segment of 


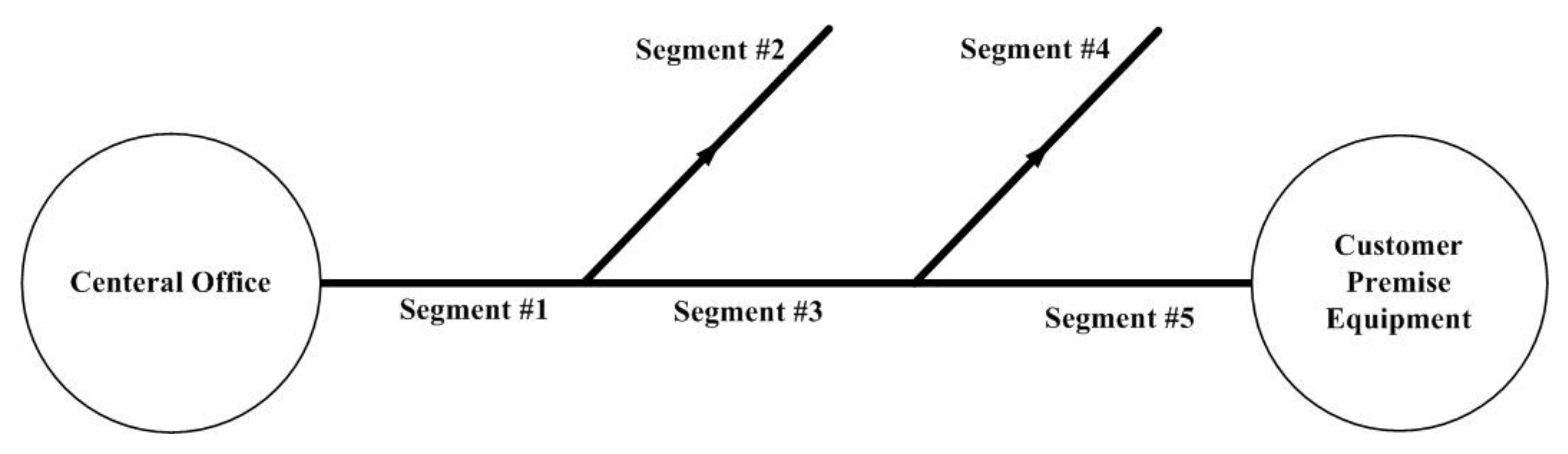

Figure 1.4: Subscriber loop with two bridged taps.

the transmission line (bridged taps and main-channel segments).

The frequency response of a subscriber loop with multiple segments can be found by multiplying the corresponding two-port $A B C D$ matrices of different segments, which are, in fact, connected in series [1]. The matrices are multiplied in the natural order of appearance from the $\mathrm{CO}$ to $\mathrm{CPE}$. As an example, for the transmission line with two bridged taps shown in Figure 1.4, one can write:

$$
\phi=\phi_{1} \cdot \phi_{2} \cdot \phi_{3} \cdot \phi_{4} \cdot \phi_{5}
$$

where $\phi_{i}$ represents the frequency response of segment $\# i, i=1, \ldots, 5$. The two-port model for the voltage source, on the other hand, is given by:

$$
\left[\begin{array}{cc}
1 & Z_{S} \\
0 & 1
\end{array}\right]
$$

where $Z_{S}$ is the source impedance. Moreover, the output voltage and current are related by the following scalar equation:

$$
V_{L}=I_{L} \cdot Z_{L}
$$


where $Z_{L}$ is the load impedance. From the above equation, one can obtain the transfer function of the transmission line as follows:

$$
H=\frac{V_{L}}{V_{S}}
$$

Now, by using the $A B C D$ model for every segment of the line, the transfer function of the subscriber loop is obtained as [1]:

$$
H=\frac{V_{L}}{V_{S}}=\frac{Z_{L}}{\left(A \cdot Z_{L}+B+C \cdot Z_{S} \cdot Z_{L}+D \cdot Z_{S}\right)}
$$

The segment numbers adopted in Figures 1.3 and 1.4 will be used hereafter to specify each segment of the line.

\subsection{Frequency-Dependency of the $R L C G$ Parameters}

The parameters $R, L, C$ and $G$ are the primary constants, namely resistance, inductance, capacitance and conductance, respectively, of the transmission line which are expressed in per unit length. These parameters also depend on frequency, and are found for twistedpairs through measurement. Practical measurements, however, are subject to error; that is why the measured $R L C G$ values may not follow smooth curves with frequency [1]. Therefore, parameterized smooth fitting models are used to represent the measured values. 


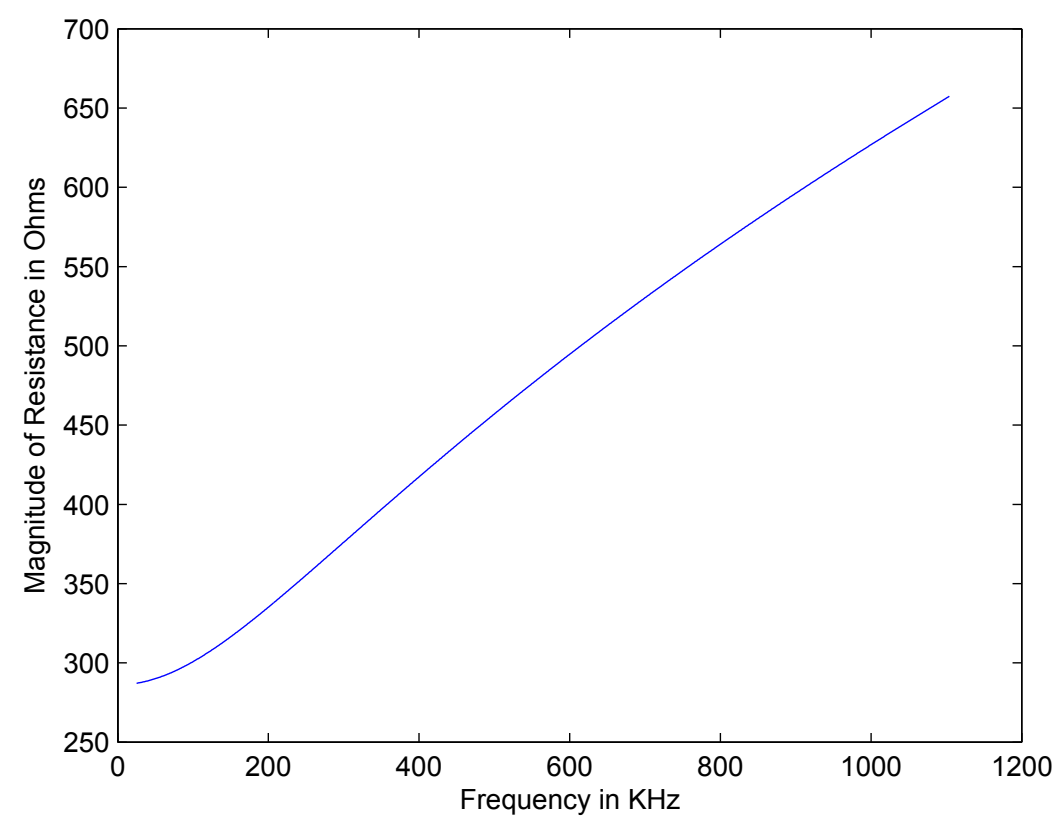

Figure 1.5: Resistance vs frequency for a 26 gauge cable.

The parameterized model for resistance with the frequency dependency is:

$$
R(f)=\frac{1}{\frac{1}{\sqrt[4]{r_{O C}^{4}+a_{C} \cdot f^{2}}}+\frac{1}{\sqrt[4]{r_{O S}^{4}+a_{S} \cdot f^{2}}}}
$$

where $r_{O C}$ and $r_{O S}$ are the copper and steel $D C$ resistances, respectively. Furthermore, $a_{C}$ and $a_{S}$ are the constants indicating the rise of resistance with frequency in the skin effect. The frequency dependency of resistance are shown in Figures 1.5 and 1.6. From these figures, it is evident that resistance is an increasing function of frequency, and at low frequencies (typically from 0 to $10 \mathrm{KHz}$ ) its value is almost constant.

The parameterized model for inductance showing the frequency dependency is:

$$
L(f)=\frac{l_{0}+l_{\infty} \cdot\left(\frac{f}{f_{m}}\right)^{b}}{1+\left(\frac{f}{f_{m}}\right)^{b}}
$$




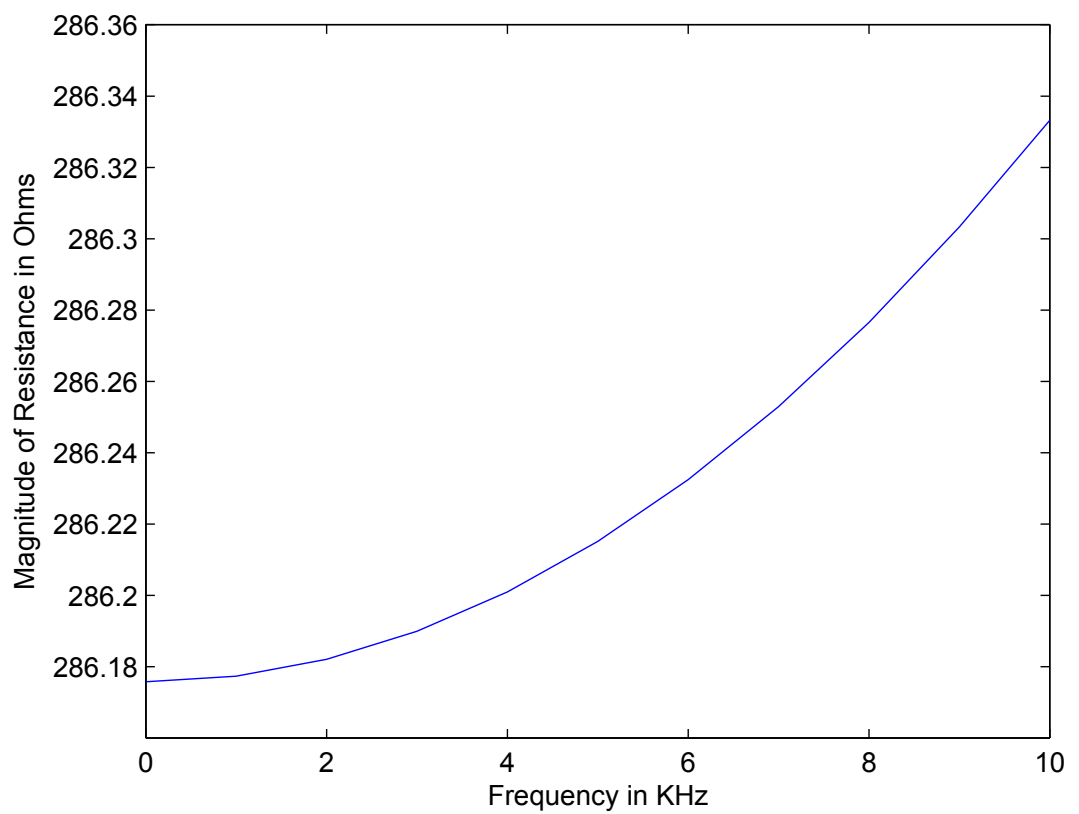

Figure 1.6: Resistance at low frequencies for a 26 gauge cable.

where $l_{0}$ and $l_{\infty}$ are the low-frequency and high-frequency inductance, respectively, and $b$ is a parameter chosen to characterize the transition between low and high frequencies in the measured inductance values. The frequency dependency of inductance can be clearly visualized from Figures 1.7 and 1.8. These figures show that inductance is a decreasing function of frequency, and at low frequencies its value is also almost constant [1]. The parameterized model for capacitance is:

$$
C(f)=c_{\infty}+c_{0} \cdot f^{-c_{e}}
$$

where $c_{\infty}$ is the contact capacitance, and $c_{0}, c_{e}$ are constants used to fit the measurement. 


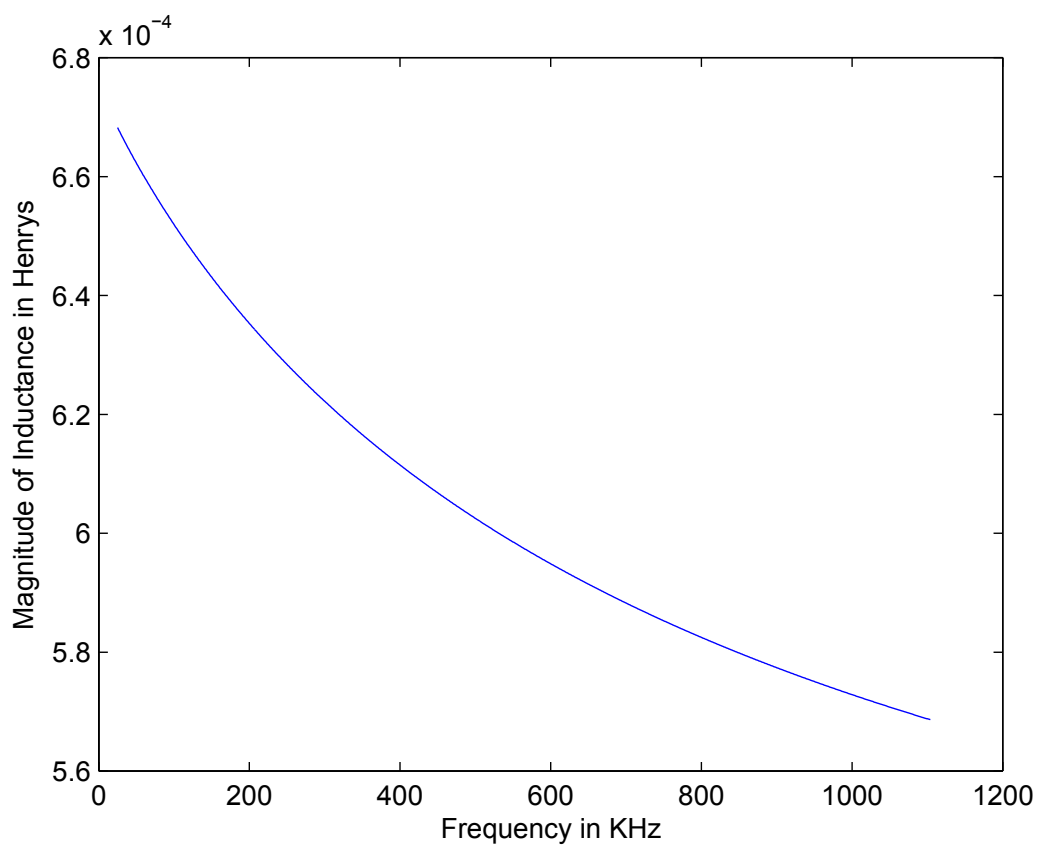

Figure 1.7: Inductance vs frequency for a 26 gauge cable.

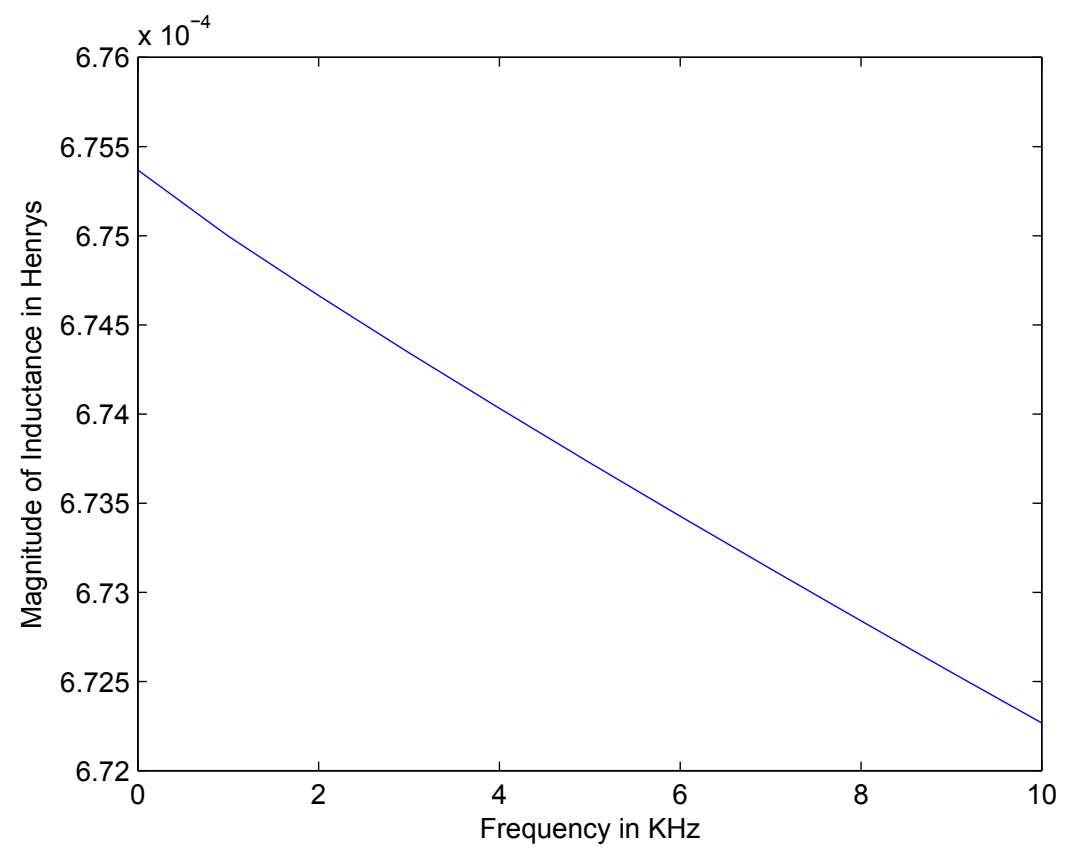

Figure 1.8: Inductance at low frequencies for a 26 gauge cable. 
The parameterized model for conductance is:

$$
G(f)=g_{0} \cdot f^{+g_{e}}
$$

where the constants $g_{0}$ and $g_{e}$ are used to fit the measurement. The parameter values for 26 and 24 gauge twisted-pairs are given in Tables 1.1 and 1.2, respectively.

Table 1.1: Parameter values for a 26 gauge twisted pair cable

\begin{tabular}{|l|c|c|c|c|}
\hline Resistance & $r_{O C}$ & $r_{O S}$ & $a_{C}$ & $a_{S}$ \\
(value) & $286.17578 \Omega / K m$ & $\infty \Omega / K m$ & 0.14769620 & 0.0 \\
Inductance & $l_{0}$ & $l_{\infty}$ & $\mathrm{b}$ & $f_{m}$ \\
(value) & $675.36888 \mu \mathrm{H} / \mathrm{Km}$ & $488.95186 \mu \mathrm{H} / \mathrm{Km}$ & 0.92930728 & $806.33863 \mathrm{KHz}$ \\
Capacitance & $C_{\infty}$ & $C_{0}$ & $C_{e}$ & - \\
(value) & $49 \mathrm{nF} / \mathrm{Km}$ & $0.0 \mathrm{nF} / \mathrm{Km}$ & $0.0 \mathrm{nF} / \mathrm{Km}$ & - \\
Conductance & $g_{0}$ & $g_{e}$ & - & - \\
(value) & $43 \mathrm{nS} / \mathrm{Km}$ & 0.70 & - & - \\
\hline
\end{tabular}

Table 1.2: Parameter values for a 24 gauge twisted pair cable

\begin{tabular}{|l|c|c|c|c|}
\hline Resistance & $r_{O C}$ & $r_{O S}$ & $a_{C}$ & $a_{S}$ \\
(value) & $174.55888 \Omega / \mathrm{Km}$ & $\infty \Omega / \mathrm{Km}$ & 0.053073481 & 0.0 \\
Inductance & $l_{0}$ & $l_{\infty}$ & $\mathrm{b}$ & $f_{m}$ \\
(value) & $617.29539 \mu \mathrm{H} / \mathrm{Km}$ & $478.97099 \mu \mathrm{H} / \mathrm{Km}$ & 1.1529766 & $553.760 \mathrm{KHz}$ \\
Capacitance & $C_{\infty}$ & $C_{0}$ & $C_{e}$ & - \\
(value) & $50 \mathrm{nF} / \mathrm{Km}$ & $0.0 \mathrm{nF} / \mathrm{Km}$ & $0.0 \mathrm{nF} / \mathrm{Km}$ & - \\
Conductance & $g_{0}$ & $g_{e}$ & - & - \\
(value) & $234.87476 \mathrm{nS} / \mathrm{Km}$ & 1.38 & - & - \\
\hline
\end{tabular}

\subsection{Objective}

The objective of the present work is to develop an algorithm to identify the full configuration of a twisted-pair transmission line from its frequency response. The algorithm can be 
used as a single-ended line testing. In other words, the line can be characterized using the information collected at the $\mathrm{CO}$ by performing a simple test. The problem of identifying the line configuration is concerned with determining each segment (main line segments and bridged taps) in terms of its length and gauge. It is assumed that the line can have up to two bridged taps. Each bridged tap divides the main line to two segments, and with two bridged taps there will be at most three segments in the main line. Furthermore, each segment of the main line and the bridged taps can have three different gauges (please see Figures 1.3 and 1.4).

\subsection{Contribution and Publication}

The problem has been solved using a proper optimization technique (which finds the closest configuration by minimizing the error in the frequency response). The core concept to solve the problem has been explained in Chapter 3. The method used to determine the lengths of the bridged taps has been explained in Chapter 2. The results of this work appeared in the following conference:

Kazi Moinul Islam, Mohammad Salehizadeh and Amir G. Aghdam, "Characterization of the Line Configuration in Wired Communication Networks," in Proceedings of ACM Research in Applied Computation Symposium (RACS), Miami, USA, November 2011. 


\section{Chapter 2}

\section{Determination of the Lengths of the}

\section{Bridged Taps and the Total Length of}

\section{the Line}

\subsection{Effects of Different Transmission Line Segments}

Optical fiber is used in modern very high speed DSL (VDSL) networks to connect the $\mathrm{CO}$ to an optical network unit (ONU). Up to about one mile of the existing copper-based telephone network infrastructure is then used from the ONU to CPE [48]. When a signal is transmitted through a twisted-pair, some of its energy leaks out from the main channel and some is reflected back to the bridging location by the open circuited twisted-pair as 
shown in Figure 2.1. The reflected signal (which is a delayed and distorted version of

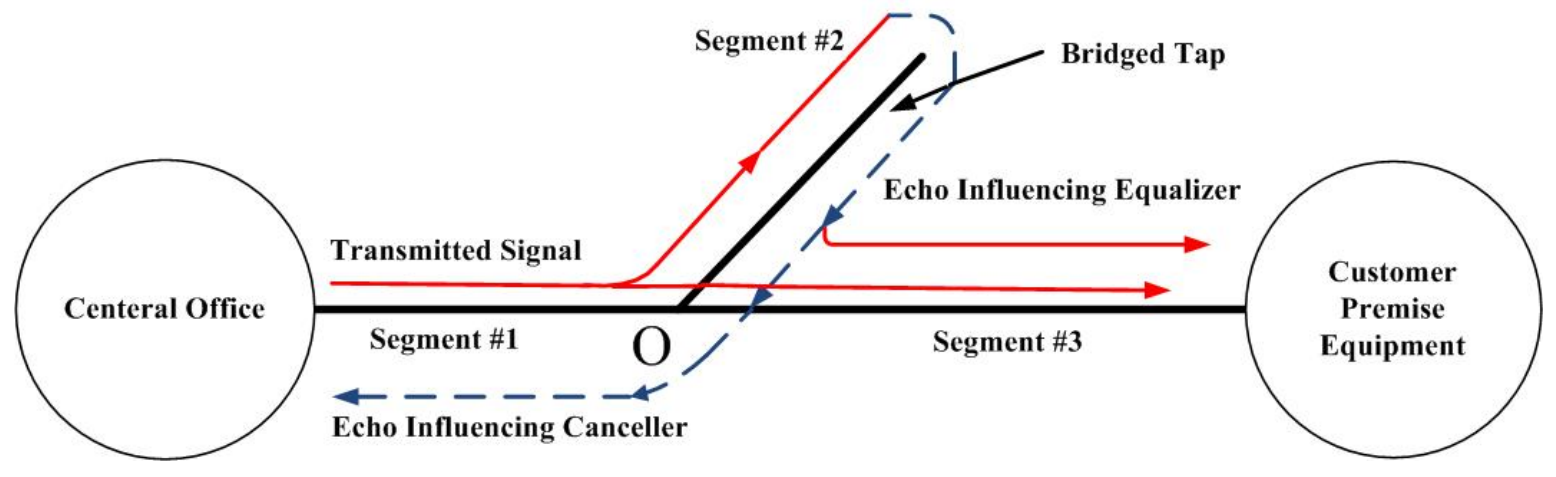

Figure 2.1: The effect of a bridged tap in the transmission line.

the main signal) creates two types of interferences as shown in the figure and discussed below:

i) The first component of interference is the one that travels in the same direction as the main signal, which is added to it and will appear as noise to the receiver.

ii) The second component of interference is the one that propagates back and will appear as an echo to the transmitter.

In addition to the above two interference components, there is another damaging effect of the bridged tap, namely net loss caused by nulls (dips) in the frequency response of the loop. In fact, for certain frequencies a sinusoidal wave reflected by the bridged tap can arrive at the bridging location with a phase shift of 180 with respect to the corresponding sinusoidal wave in the main signal. When this occurs, the two frequency components subtract in amplitude and the result is a noticeable dip in the overall magnitude response of the loop around this frequency. These dips occur at frequencies for which the bridged tap 
length $d_{b t}$ is equal to an odd multiple of one quarter of the wavelength of that frequency. Let $v(f)=\frac{1}{\tau_{\phi}(f)}$ and $\lambda(f)=\frac{v(f)}{f}$, where $v(f)$ is the phase velocity, $\tau_{\phi}(f)$ is the phase delay, and $\lambda(f)$ is the wavelength. The first null occurs at [5]:

$$
f_{0}=\frac{v\left(f_{0}\right)}{\lambda\left(f_{0}\right)}=\frac{v\left(f_{0}\right)}{4 d_{b t}}=\frac{1}{4 d_{b t} \tau_{\phi}\left(f_{0}\right)}
$$

Other nulls occur at the following frequencies [5]:

$$
f=(2 j+1) f_{0}, \quad j=1,2,3, \ldots
$$

The expression (2.1) is highly nonlinear; however, it can be solved by approximation for bridged taps up to a certain length (less than $2 \mathrm{Kft}$ ). In such a configuration, the nulls occur in a frequency range where the phase delay is approximately constant and equal to about 8.7 micro sec/mile [5]. This leads to the following equality:

$$
d_{b t}=\frac{150}{f_{0}}
$$

where $f_{0}$ and $d_{b t}$ are in $\mathrm{KHz}$ and $\mathrm{Kft}$, respectively.

\subsection{Determine the Lengths of the Bridged Taps}

Figure 2.2 depicts the magnitude response of a transmission line with one bridged tap, where the segments are characterized as: 
Segment \#1 (CO-side of the main channel): $2500 \mathrm{ft}, 26$ gauge

Segment \#2 (bridged tap): $500 \mathrm{ft}, 24$ gauge

Segment \#3 (CPE-side of the main channel): $1500 \mathrm{ft}, 24$ gauge

This figure shows the first null occurs approximately at $304 \mathrm{KHz}$ (and the second one at

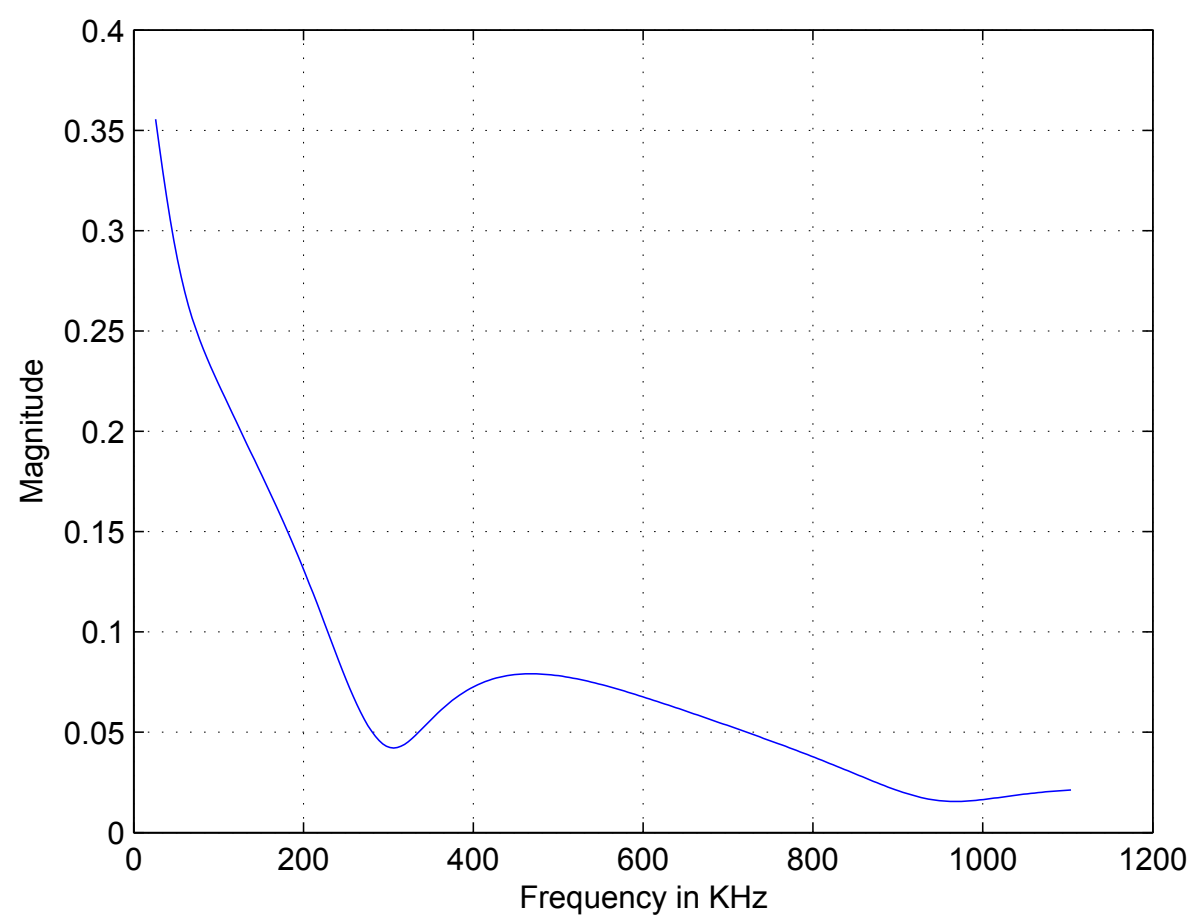

Figure 2.2: The magnitude response of a loop with single bridged tap.

around $912 \mathrm{KHz}$, which is equal to $304 \times 3$ ). Thus, from equation (2.3) the length of the bridged tap is obtained to be $494 \mathrm{ft}$, which is very close to the exact value.

By generalizing the method presented in the previous section, the lengths of multiple bridged taps in a loop can be heuristically determined from equation (2.3). First of all, 
one needs to identify distinct groups of dips which meet the condition expressed by equation (2.2). The number of such groups is, in fact, the number of bridged taps in the loop. The length of each bridged tap can then be obtained from the smallest frequency in each group. However, such groupings can be made only if the length of a bridged tap is not an odd multiple of the length of another bridged tap (because in such cases the corresponding nulls coincide). Throughout this work, it is assumed that the length of a bridged tap is not an odd multiple of the length of another bridged tap (note that the length of a line segment is by assumption nonzero).

As an example, the magnitude response of a transmission line with two bridged taps

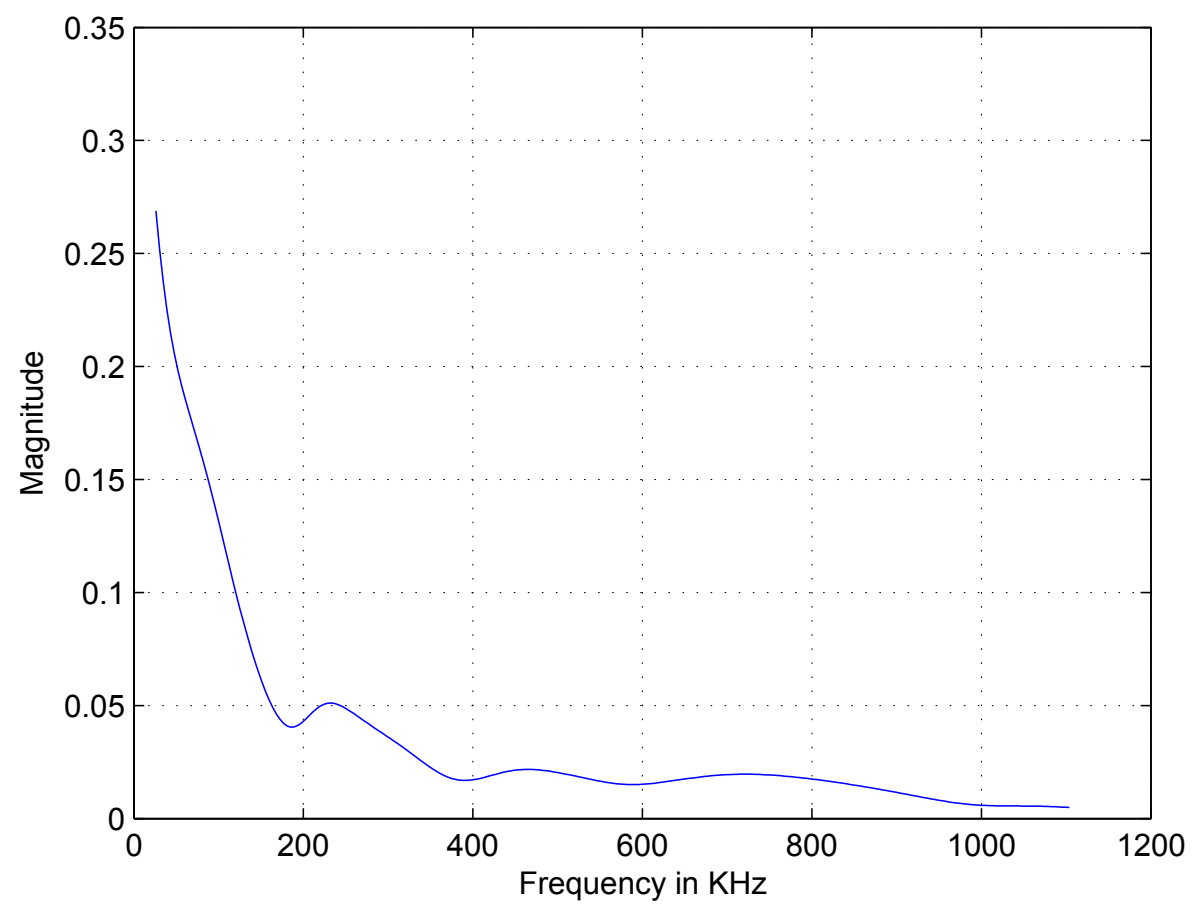

Figure 2.3: The magnitude response of a loop with two bridged taps.

is given in Figure 2.3. The smallest null frequencies corresponding to these bridged taps 
are $183 \mathrm{KHz}$ and $390 \mathrm{KHz}$. Hence, from equation (2.3) the bridged tap lengths are obtained as $818 \mathrm{ft}$ and $385 \mathrm{ft}$.

\subsection{A Method to Determine the Total Length of the Trans- mission Line}

As shown in the previous section, the frequency response of a twisted-pair is a gradually decaying function of frequency. It can be observed from Figure 2.2 that there are some oscillations in the curve at high frequencies, which is, in fact, due to the presence of the bridged tap. However, the curve is smooth at low frequencies, which contains very important information. An estimation of the total line length can be made by fitting the low-frequency curve with a smooth curve. Detailed procedure for this is given below in a step-by-step manner.

i) Step 1: Fit the magnitude response with a polynomial of sufficiently high degree.

ii) Step 2: To obtain a rough estimate of the total line length, it is assumed that there is no bridged tap connection. The frequency response of the simplified line is approximated subsequently by applying the two-port network model of communication line as described in Chapter 1. The frequency response is a function of frequency with unknown line length, i.e.:

$$
H=g(f, d)
$$

where $f$ is the frequency and $d$ is the total length of the line. 
iii) Final step: Now, there are two equations: one from the best fitted curve (which is a function of frequency) and the other from the simplified line applying the two-port network model. An estimate of the line length can now be obtained by using a proper optimization procedure, e.g., Nelder-Mead's simplex search method.

\subsubsection{Simulation Result for Initial Length Estimate of the Main Line}

In this subsection two different line configurations with two bridged taps are considered: one with relatively short segments, and one with longer segments. The procedure given earlier will be used to estimate the total length of the main line.

Configuration 1: Consider a wired communication line with the following characteristics (see Figure 1.4 for segment numbers):

Segment \#1: $1500 \mathrm{ft}, 26$ gauge

Segment \#2: $700 \mathrm{ft}, 22$ gauge

Segment \#3: $1000 \mathrm{ft}, 24$ gauge

Segment \#4: $400 \mathrm{ft}, 22$ gauge

Segment \#5: 500 ft, 22 gauge

Note that the total length of the main line in this configuration (segments \#1, 3, 5) is 3

Kft. The magnitude response of this line along with the $10^{\text {th }}$-order fitting polynomial for that obtained by MATLAB is given in Figure 2.4. Now, using the algorithm provided in Section 2.3, the total length of the main line is estimated to be $3.0412 \mathrm{Kft}$, which is very 


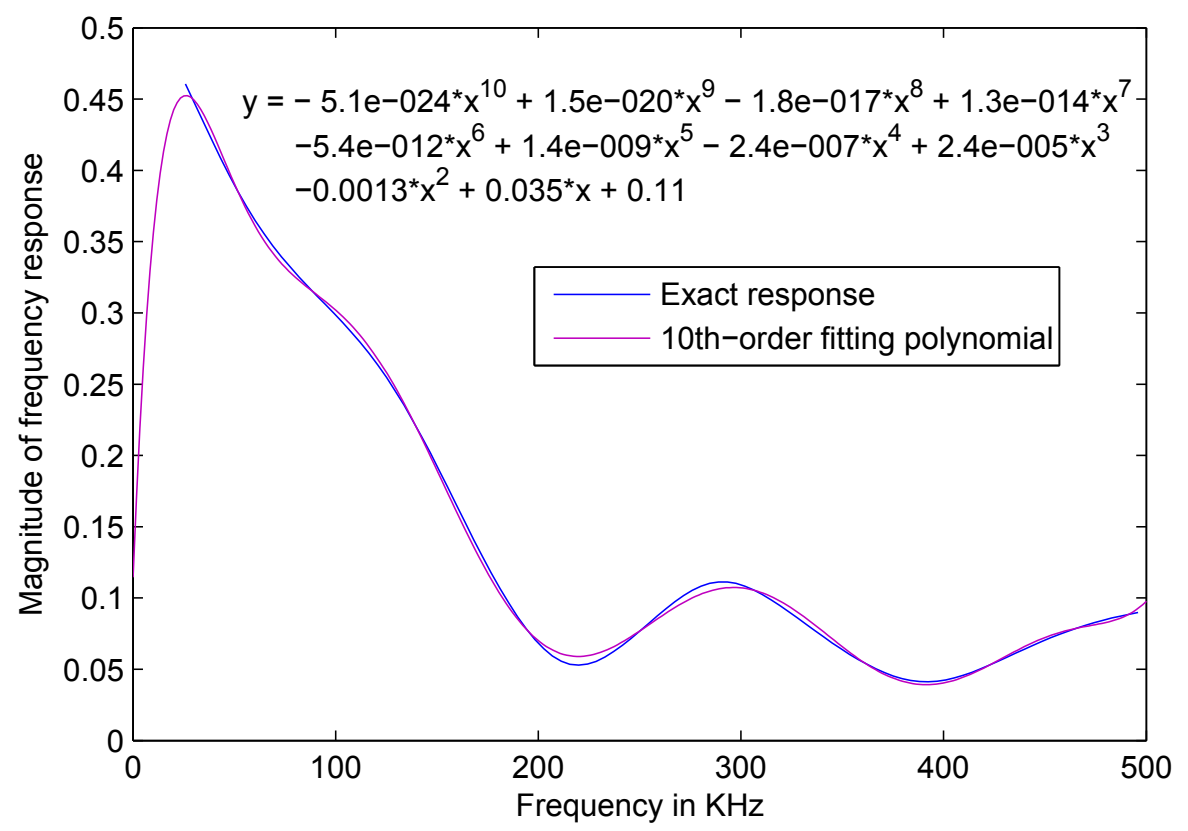

Figure 2.4: The real magnitude response and the associated fitting polynomial of order 10 for Configuration 1.

close to the exact line length.

Configuration 2: Consider now a line configuration with longer segments as follows:

Segment \#1: $4000 \mathrm{ft}, 26$ gauge

Segment \#2: $1500 \mathrm{ft}, 24$ gauge

Segment \#3: $2500 \mathrm{ft}, 24$ gauge

Segment \#4: $1000 \mathrm{ft}, 24$ gauge

Segment \#5: $1500 \mathrm{ft}, 22$ gauge

The magnitude response of this line is provided in Figure 2.5 together with the corresponding $10^{\text {th }}$-order fitting polynomial . Using the algorithm given in Section 2.3, an 


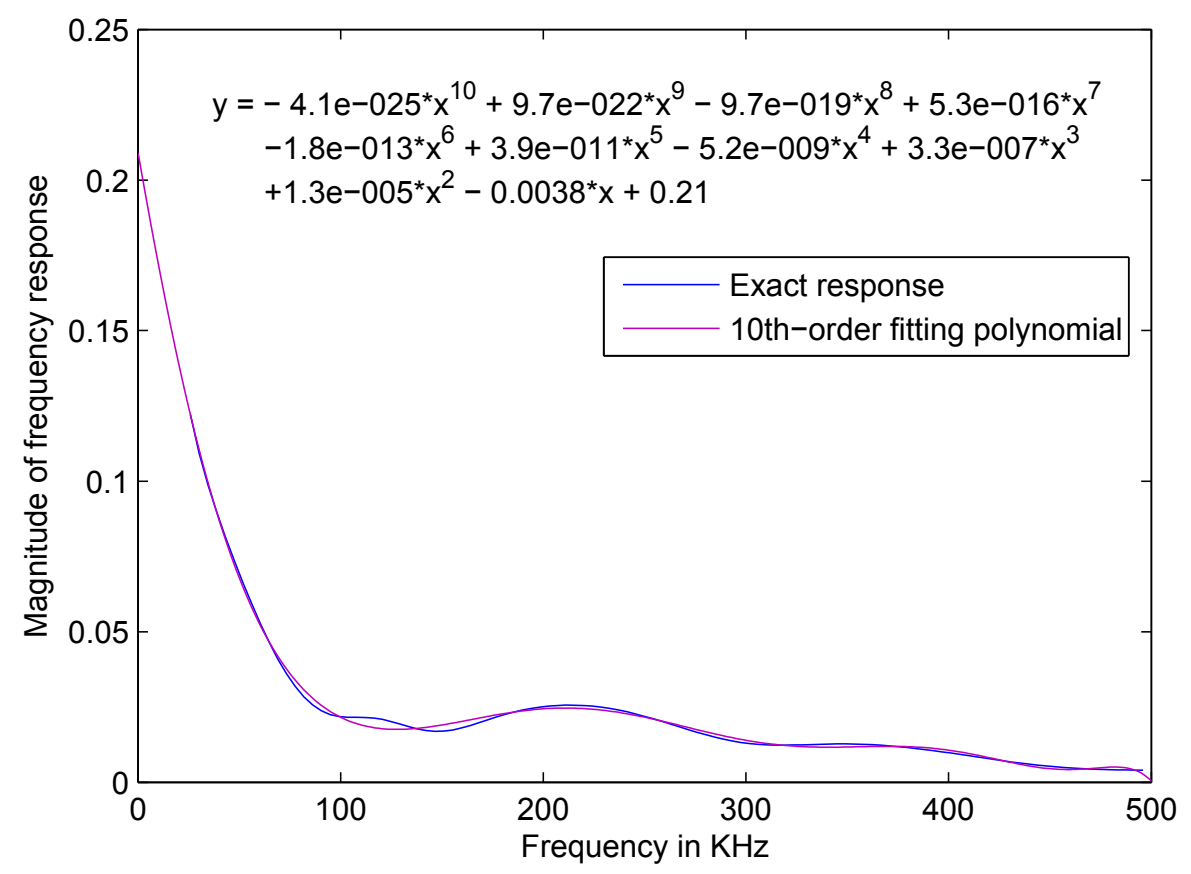

Figure 2.5: The real magnitude response and the associated fitting polynomial of order 10 for Configuration 2.

estimate of the total length of the main line is obtained to be $8.0297 \mathrm{Kft}$, which means the estimation error in this case is $0.371 \%$.

\subsection{Frequency-Dependency of the $R L C G$ Parameters}

Any technique used to identify the line configuration should take the frequency dependency of the line model, namely resistance $R$, inductance $L$, capacitance $C$ and conductance $G$, into account. This dependency is not the same for different line gauges, as noted earlier. In this thesis, the frequency dependency of the parameters of the transmission line is taken into consideration in the optimization procedure introduced in the next chapter. 
To improve the speed of the procedure, look-up tables will be used to compute the frequency response of the line in the underlying procedure. The look-up tables for the three line gauges are given in Tables 2.1-2.3. 
Table 2.1: $R L G C$ values for a 26 gauge twisted pair cable

\begin{tabular}{|c|c|c|c|c|}
\hline Frequency(Hertz) & $\mathrm{R}(\mathrm{Ohms} / \mathrm{mile})$ & L(Henrys/mile) & G(Siemens/mile) & $\mathrm{C}($ Farads/mile $)$ \\
\hline 1 & 440.75 & 0.9861 & 0.0 & 0.083 \\
\hline 5 & 440.75 & 0.9861 & 0.001 & 0.083 \\
\hline 10 & 440.75 & 0.9861 & 0.002 & 0.083 \\
\hline 15 & 440.76 & 0.9861 & 0.003 & 0.083 \\
\hline 20 & 440.76 & 0.9861 & 0.004 & 0.083 \\
\hline 30 & 440.76 & 0.9861 & 0.005 & 0.083 \\
\hline 50 & 440.76 & 0.9861 & 0.008 & 0.083 \\
\hline 70 & 440.76 & 0.9861 & 0.011 & 0.083 \\
\hline 100 & 440.76 & 0.9861 & 0.016 & 0.083 \\
\hline 150 & 440.76 & 0.9861 & 0.022 & 0.083 \\
\hline 200 & 440.76 & 0.9860 & 0.028 & 0.083 \\
\hline 300 & 440.76 & 0.9860 & 0.040 & 0.083 \\
\hline 500 & 440.77 & 0.9859 & 0.063 & 0.083 \\
\hline 700 & 440.78 & 0.9859 & 0.084 & 0.083 \\
\hline 1000 & 440.79 & 0.9858 & 0.115 & 0.083 \\
\hline 1500 & 440.81 & 0.9856 & 0.164 & 0.083 \\
\hline 2000 & 440.83 & 0.9854 & 0.210 & 0.083 \\
\hline 3000 & 440.88 & 0.9850 & 0.299 & 0.083 \\
\hline 5000 & 441.01 & 0.9843 & 0.466 & 0.083 \\
\hline 7000 & 441.15 & 0.9836 & 0.625 & 0.083 \\
\hline 10000 & 441.39 & 0.9825 & 0.853 & 0.083 \\
\hline 15000 & 441.87 & 0.9807 & 1.213 & 0.083 \\
\hline 20000 & 442.88 & 0.9789 & 1.558 & 0.083 \\
\hline 30000 & 443.88 & 0.9753 & 2.217 & 0.083 \\
\hline 50000 & 447.81 & 0.9660 & 3.458 & 0.083 \\
\hline 70000 & 453.09 & 0.9546 & 4.634 & 0.083 \\
\hline 100000 & 463.39 & 0.9432 & 6.320 & 0.083 \\
\hline 150000 & 485.80 & 0.9306 & 8.993 & 0.083 \\
\hline 200000 & 513.04 & 0.9212 & 11.550 & 0.083 \\
\hline 300000 & 575.17 & 0.9062 & 16.436 & 0.083 \\
\hline 500000 & 699.61 & 0.8816 & 25.633 & 0.083 \\
\hline 700000 & 812.95 & 0.8614 & 34.351 & 0.083 \\
\hline 1000000 & 956.65 & 0.8381 & 46.849 & 0.083 \\
\hline 1500000 & 1154.38 & 0.8146 & 66.665 & 0.083 \\
\hline 2000000 & 1321.07 & 0.8001 & 85.624 & 0.083 \\
\hline 3000000 & 1600.68 & 0.7823 & 121.841 & 0.083 \\
\hline 5000000 & 2044.07 & 0.7638 & 190.021 & 0.083 \\
\hline
\end{tabular}


Table 2.2: $R L G C$ values for a 24 gauge twisted pair cable

\begin{tabular}{|l|c|c|c|c|}
\hline Frequency(Hertz) & R(Ohms/mile) & L(Henrys/mile) & G(Siemens/mile) & C(Farads/mile) \\
\hline 1 & 277.19 & 0.9861 & 0.0 & 0.083 \\
5 & 277.19 & 0.9861 & 0.0010 .083 & \\
10 & 277.19 & 0.9861 & 0.002 & 0.083 \\
15 & 277.19 & 0.9861 & 0.003 & 0.083 \\
20 & 277.19 & 0.9861 & 0.004 & 0.083 \\
30 & 277.19 & 0.9861 & 0.005 & 0.083 \\
50 & 277.19 & 0.9861 & 0.008 & 0.083 \\
70 & 277.19 & 0.9861 & 0.011 & 0.083 \\
100 & 277.19 & 0.9861 & 0.016 & 0.083 \\
150 & 277.20 & 0.9860 & 0.022 & 0.083 \\
200 & 277.20 & 0.9860 & 0.028 & 0.083 \\
300 & 277.20 & 0.9860 & 0.040 & 0.083 \\
500 & 277.21 & 0.9859 & 0.063 & 0.083 \\
700 & 277.22 & 0.9858 & 0.084 & 0.083 \\
1000 & 277.23 & 0.9857 & 0.115 & 0.083 \\
1500 & 277.25 & 0.9854 & 0.164 & 0.083 \\
2000 & 277.28 & 0.9852 & 0.210 & 0.083 \\
3000 & 277.34 & 0.9848 & 0.299 & 0.083 \\
5000 & 277.48 & 0.9839 & 0.466 & 0.083 \\
7000 & 277.66 & 0.9829 & 0.625 & 0.083 \\
10000 & 277.96 & 0.9816 & 0.853 & 0.083 \\
15000 & 278.58 & 0.9793 & 1.213 & 0.083 \\
20000 & 279.35 & 0.9770 & 1.558 & 0.083 \\
30000 & 281.30 & 0.9723 & 2.217 & 0.083 \\
50000 & 286.82 & 0.9577 & 3.458 & 0.083 \\
70000 & 294.29 & 0.9464 & 4.634 & 0.083 \\
100000 & 308.41 & 0.9347 & 6.320 & 0.083 \\
150000 & 337.22 & 0.9204 & 8.993 & 0.083 \\
200000 & 369.03 & 0.9087 & 11.550 & 0.083 \\
300000 & 431.55 & 0.8885 & 16.436 & 0.083 \\
500000 & 541.69 & 0.8570 & 25.633 & 0.083 \\
700000 & 632.08 & 0.8350 & 34.351 & 0.083 \\
1000000 & 746.04 & 0.8146 & 46.849 & 0.083 \\
1500000 & 902.84 & 0.7947 & 66.665 & 0.083 \\
\hline & & & & \\
\hline
\end{tabular}


Table 2.3: $R L G C$ values for a 22 gauge twisted pair cable

\begin{tabular}{|l|c|c|c|c|}
\hline Frequency(Hertz) & R(Ohms/mile) & L(Henrys/mile) & G(Siemens/mile) & C(Farads/mile) \\
\hline 1 & 174.27 & 0.9861 & 0.000 & 0.083 \\
5 & 174.27 & 0.9861 & 0.001 & 0.083 \\
10 & 174.27 & 0.9861 & 0.001 & 0.083 \\
15 & 174.27 & 0.9861 & 0.001 & 0.083 \\
20 & 174.27 & 0.9861 & 0.002 & 0.083 \\
30 & 174.27 & 0.9861 & 0.003 & 0.083 \\
50 & 174.27 & 0.9861 & 0.005 & 0.083 \\
70 & 174.27 & 0.9861 & 0.006 & 0.083 \\
100 & 174.27 & 0.9861 & 0.009 & 0.083 \\
150 & 174.27 & 0.9860 & 0.013 & 0.083 \\
200 & 174.27 & 0.9860 & 0.017 & 0.083 \\
300 & 174.28 & 0.9860 & 0.024 & 0.083 \\
500 & 174.29 & 0.9858 & 0.040 & 0.083 \\
700 & 174.29 & 0.9857 & 0.054 & 0.083 \\
1000 & 174.31 & 0.9856 & 0.076 & 0.083 \\
1500 & 174.34 & 0.9853 & 0.110 & 0.083 \\
2000 & 174.37 & 0.9850 & 0.145 & 0.083 \\
3000 & 174.44 & 0.9844 & 0.211 & 0.083 \\
5000 & 174.62 & 0.9833 & 0.341 & 0.083 \\
7000 & 174.83 & 0.9821 & 0.467 & 0.083 \\
10000 & 175.22 & 0.9804 & 0.652 & 0.083 \\
15000 & 176.06 & 0.9778 & 0.954 & 0.083 \\
20000 & 177.11 & 0.9744 & 1.248 & 0.083 \\
30000 & 179.86 & 0.9672 & 1.824 & 0.083 \\
50000 & 187.64 & 0.9491 & 2.943 & 0.083 \\
70000 & 197.71 & 0.9372 & 4.032 & 0.083 \\
100000 & 215.55 & 0.9237 & 5.630 & 0.083 \\
150000 & 247.57 & 0.9055 & 8.229 & 0.083 \\
200000 & 277.95 & 0.8898 & 10.772 & 0.083 \\
300000 & 333.39 & 0.8642 & 15.744 & 0.083 \\
500000 & 421.57 & 0.8309 & 25.396 & 0.083 \\
700000 & 493.24 & 0.8123 & 34.796 & 0.083 \\
1000000 & 583.59 & 0.7950 & 48.587 & 0.083 \\
1500000 & 707.91 & 0.7783 & 71.014 & 0.083 \\
\hline
\end{tabular}




\section{Chapter 3}

\section{Identification of Line Configuration}

\subsection{Finding an Initial Estimate for Line Configuration}

Given the frequency response (magnitude only) of a transmission line, it is desired now to characterize its configuration by finding the length and gauge of each segment of the line. To this end, an optimization procedure is developed which aims to find a configuration with the closest magnitude response to the given function (in the sense of integral of the squared error in the frequency domain). As the first step of the underlying optimization procedure, it is important to find an initial estimate of the length of each segment within about $20 \%$ accuracy. If the initial estimate is not in a reasonable neighborhood of the exact values, the algorithm can converge to different local minima, or even diverge. The following assumptions will be used in the development of the procedure. 
Assumption 1: The length of each bridged taps is assumed to be either 0 or between $400 \mathrm{ft}$ and $1500 \mathrm{ft}$.

Assumption 2: There can be only one or two bridged taps. In the latter case, both bridged taps have the same gauge.

Assumption 3: The overall length of the main channel does not exceed $20 \mathrm{Kft}$.

Assumption 4: The frequency response of the line is given in a bin-by-bin basis (each frequency bin is $4.3125 \mathrm{KHz})$, from the 6th bin $(25.875 \mathrm{KHz})$ to the 256th bin $(1.104$ $\mathrm{MHz})$.

It is to be noted that Assumption 1-3 are in accordance with the standard loop configurations [4]. Assumption 4, on the other hand, reflects the practical constraints in measuring the frequency response of the line from the CO. To obtain an initial estimate for the length of the bridged tap, the frequency of the first null caused by each bridged tap in the frequency response will be used in equation 2.3, as discussed in Chapter 2. To check different line configurations, 5 line segments are considered as the most general case (two bridged taps and three main line segments). Note that the case of one bridged tap or no bridged tap in the configuration, are special cases of a 5-segment line, where the length of one or two segments is zero. The number of different combinations of line segments which one needs to check is as follows:

i) Each segment can have 3 different gauges. Since both bridged taps are assumed to 
have identical gauges, the total number of possible gauge combinations for the five segments is equal to $3^{4}=81$ [29].

ii) In order to obtain a reasonably accurate initial estimate, the main line will be divided to 12 units. It is straightforward to verify that the total number of different combinations for this units (corresponding to a line with two bridged taps) is $1+2+\ldots+12=78[29]$. Note that the real length of each unit can be obtained by dividing the overall length of the channel by 12 . Note also that using the above technique the minimum number of units for a segment is zero, and the maximum number for it is 12 .

The initial configuration search is accomplished by a MATLAB code which finds the frequency response of a total of $81 \times 78=6318$ combinations in a bin-by-bin format. The difference between the magnitude of this frequency response and that of the given frequency response is then computed as a measure of error (integral of the squared error

over frequency). The combination corresponding to the minimum error will be chosen as the initial condition for the optimization procedure.

\subsection{Search for the Optimum Configuration}

The initial line parameters generated in the previous stage will now be used in the main optimization program to find the closest match for the line configuration. The performance index is the numerical integral of the squared error (the difference between the 
magnitude responses of the real line and the configuration considered by the optimization program in each iteration). The program uses Nelder-Mead's simplex search method [20]-[27], and examines different line gauges for different segments in a way similar to the initialization program. If the performance index resulted by the optimization program for a specific configuration is less than a reasonable margin, the program will stop and the corresponding configuration will be the final result.

The problem solved here is, in fact, a constrained optimization problem, as the length of each segment must be positive, and by assumption, should not exceed certain limits. These constraints are implemented by considering a penalty function in the corresponding MATLAB code. On the other hand, in order to avoid long execution times, reasonable limits have been considered for the number of iterations and also for the accuracy of the results in the optimization procedure, as the termination conditions.

Remark 1: If the initial estimates provided to the main optimization program are too far from the exact values, they may not lie in the global optimum's domain of attraction in which case the algorithm does not converge to the desired configuration as noted before. This shows the importance of the initialization phase. A similar problem also occurs when the real line configuration does not satisfy required assumptions (e.g., has more than five segments). In any case, the algorithm returns a configuration with the closest frequency response to the real line. 


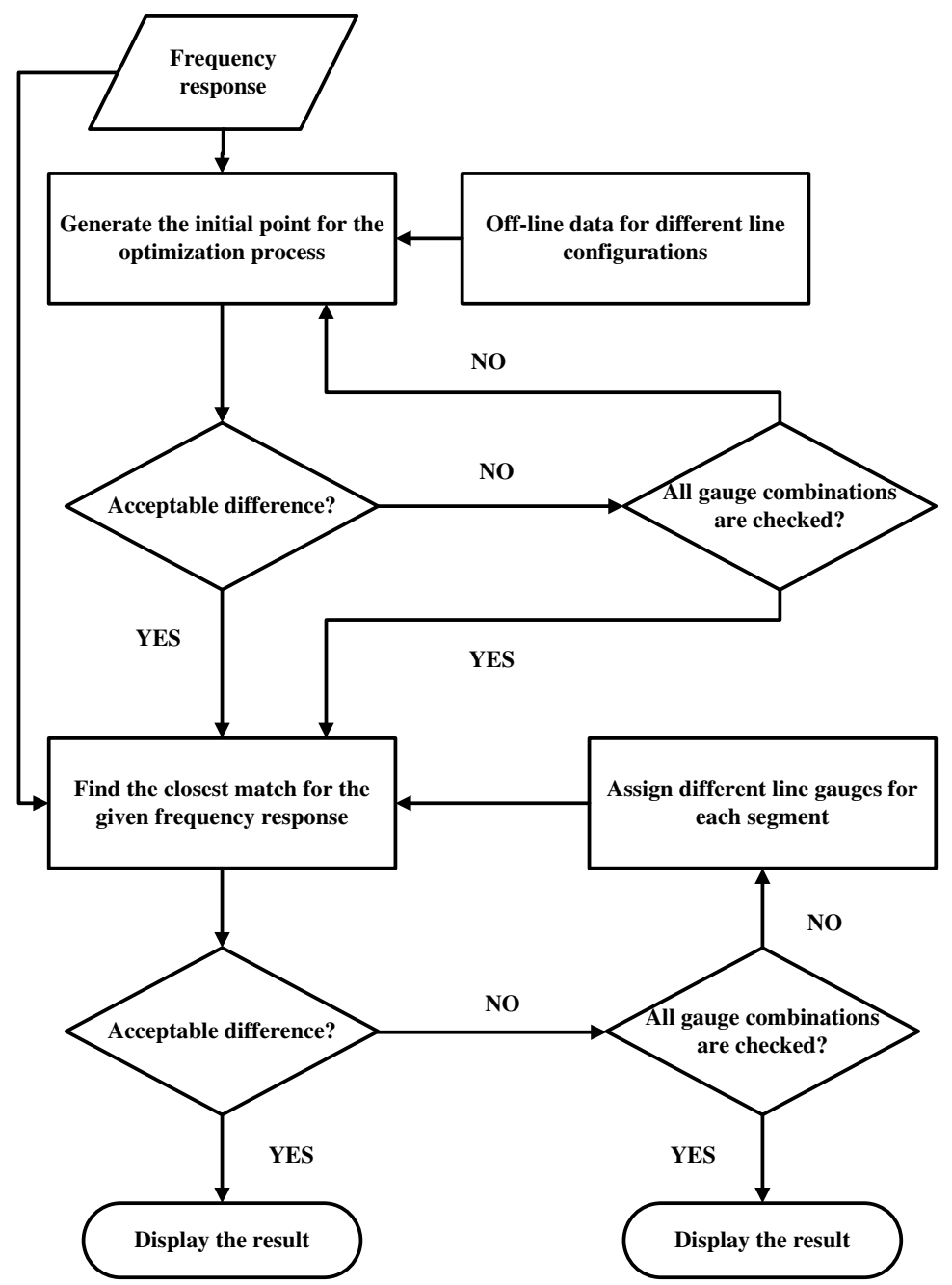

Figure 3.1: The flowchart of the proposed algorithm. 


\subsection{Reducing Program Execution Time}

An important practical issue in the line identification program is the execution time. Due to the complexity of the equations, it can take up to a few weeks for the whole program to run for a typical line with two bridged taps. Using different tricks, however, one can reduce the execution time to about 30 minutes. To accomplish this, the following steps are taken:

i) The MATLAB code generating the frequency response of the transmission line was written in a way to avoid repeated time consuming command. This reduced the execution time about four times.

ii) Some of MATLAB commands in the optimization program were written to avoid several function callings. This reduced the execution time about two times.

iii) By experiment, the most informative points in frequency were identified. By computing the frequency response and error index over the identified points of frequency, the overall execution time was reduced to about $70 \%$ of the normal execution time.

iv) Several programs were written to generate off line data, which can be used in the main program to save some time in online computation. It takes about 10 days for the off line data to be generated and it saves almost the same amount of time in the 
online algorithm.

\subsection{MATLAB Programs}

The main programs are called initialLineMatch and finalLineMatch and the data files are length_coef, lengthSimulationData_22, lengthSimulationData_24 and lengthSimulationData_26. The data files are generated by lengthSimulation and initialEstimateTest_10000 programs. The total number of lines written in $M A T L A B$ is about 1200 lines for the main programs and 950 lines for the data generating codes. The steps of the program execution are given in the following subsections.

\subsubsection{Steps or Pseudocode of the Initialization Phase}

This program obtains an initial estimate of the line configuration. This estimate needs to be sufficiently close to the exact configuration in order for the optimization procedure (in the next phase) converge. The steps of the algorithm are given below:

i) Finding the initial estimate for the bridged tap lengths.

ii) Using part of the low-frequency axis which is more informative. 
iii) Loading the primary constants $(R L G C)$ files for the frequency response generator program corresponding to different gauges.

iv) Loading the restored values from the data files for different lengths and gauges corresponding to different bridged tap gauges.

v) Choosing the lines corresponding to the approximately calculated bridged tap lengths.

vi) Finding the initial estimate for the line length (one estimate for each combination of bridged tap lengths), considering segments with different gauges and different lengths in the main line.

vii) Rearranging the gauges of the main line segments.

viii) Rearranging the gauges of the bridged taps.

ix) Generating the output of the initialization phase.

\subsubsection{Steps or Pseudocode of the Optimization Phase}

This program finds the closest line configuration for a given frequency response. It checks three different line gauges $(22,24,26)$ for each segment of a line with one or two bridged 
taps. The steps of the optimization phases are as follow:

i) Getting data for different gauge combinations.

ii) Setting the optimization Parameters.

iii) Defining the function that calculates the error to be minimized.

iv) Setting up a simplex near the initial guess. Iteration takes place until the diameter of the simplex is less than a prescribed termination tolerance value, and the function values are sufficiently close to zero (within a prescribed error margin) or the max function evaluations are exceeded.

v) Using the fminsearch function to get the configuration with minimum error.

vi) Providing the optimum configuration for the given frequency response. 


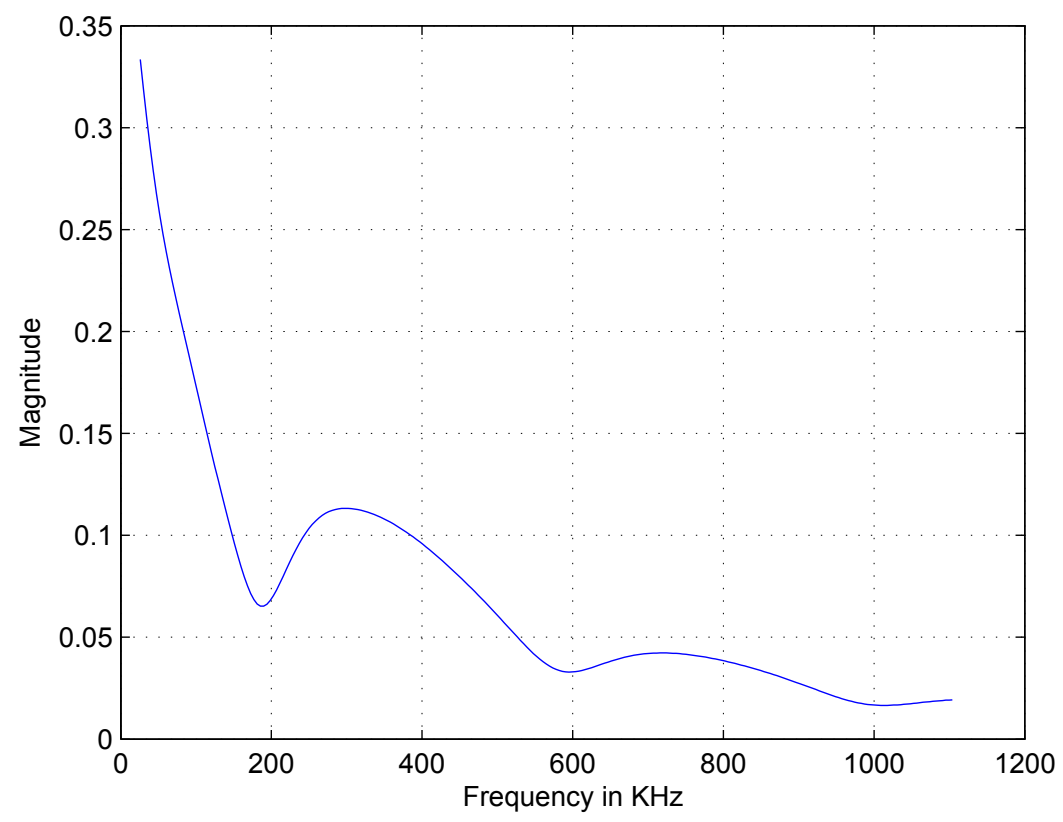

Figure 3.2: Magnitude response for the transmission line of Example 1.

\subsection{Simulation Results}

Four examples are given in this section for the characterization of a transmission line from the bin-by-bin magnitude response. In the first example a transmission line with one bridged tap and in the second example a transmission line with two bridged taps is considered.

Example 1: Consider a transmission line with three segments as follows (for segment numbers see Figure 1.3):

Segment \#1: 3000 ft, 26 gauge

Segment \#2: $800 \mathrm{ft}, 24$ gauge 
Segment \#3: $1000 \mathrm{ft}, 24$ gauge

The magnitude response of this transmission line is shown in Figure 3.2, and the corresponding information is provided in a bin-by-bin fashion to the initialization and optimization procedures developed in this work. The outcome of the initialization procedure is as follows (for segment numbers see Figure 1.4):

Segment \#1: $2689 \mathrm{ft}, 26$ gauge

Segment \#2: $768 \mathrm{ft}, 26$ gauge

Segment \#3: $672.25 \mathrm{ft}, 26$ gauge

Segment \#4: $0.0 \mathrm{ft}, 26$ gauge

Segment \#5: $672.25 \mathrm{ft}, 22$ gauge

It can be verified that maximum discrepancy of the initial estimates from the exact values is within $20 \%$. These values are subsequently provided to the main optimization procedure, which arrives at the following values:

Segment \#1: 3002 ft, 26 gauge

Segment \#2: $800 \mathrm{ft}, 24$ gauge

Segment \#3: $996 \mathrm{ft}, 24$ gauge

This demonstrates that the procedure has converged to the exact values for this configuration. The initialization and optimization procedures for this example take $2.20 \mathrm{~min}$ and $1.38 \mathrm{~min}$, respectively, on a personal computer with a $2.33 \mathrm{GHz}$ Intel Core 2 Duo CPU and 2.00 GB RAM. 


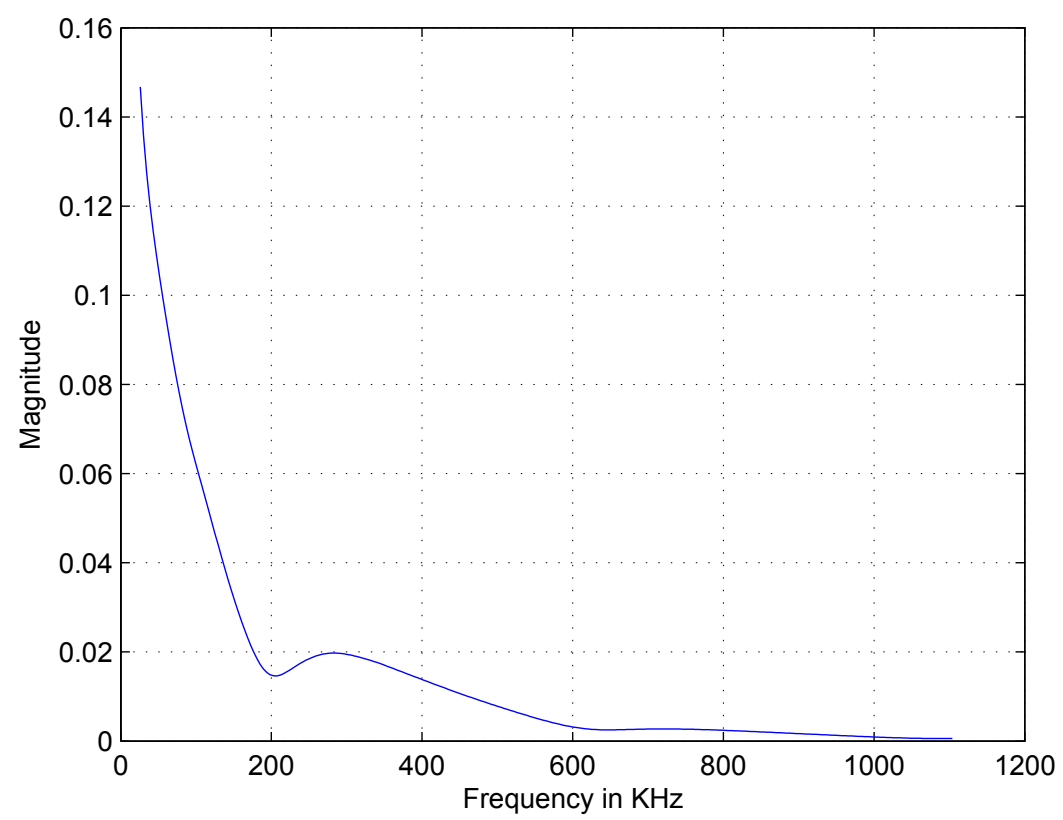

Figure 3.3: Magnitude response for the transmission line of Example 2.

Example 2: Consider a transmission line with three segments as follows (for segment numbers see Figure 1.3):

Segment \#1: $4050 \mathrm{ft}, 26$ gauge

Segment \#2: $750 \mathrm{ft}, 24$ gauge

Segment \#3: 5042 ft, 22 gauge

The magnitude response of this transmission line is shown in Figure 3.3, and the corresponding information is provided in a bin-by-bin fashion to the initialization and optimization procedures developed in this work. The outcome of the initialization procedure is as follows (for segment numbers see Figure 1.4): 
Segment \#1: $3161.5 \mathrm{ft}, 26$ gauge

Segment \#2: $768 \mathrm{ft}, 24$ gauge

Segment \#3: $3161.5 \mathrm{ft}, 26$ gauge

Segment \#4: $0.0 \mathrm{ft}, 26$ gauge

Segment \#5: 393.2 ft, 22 gauge

It can be verified that maximum discrepancy of the initial estimates from the exact values is within $20 \%$. These values are subsequently provided to the main optimization procedure, which arrives at the following values:

Segment \#1: $4050 \mathrm{ft}, 26$ gauge

Segment \#2: $750 \mathrm{ft}, 24$ gauge

Segment \#3: $5041 \mathrm{ft}, 22$ gauge

This demonstrates that the procedure has converged to the exact values for this configuration. The initialization and optimization procedures for this example take $2.20 \mathrm{~min}$ and $1.2 \mathrm{~min}$, respectively, on a personal computer with a $2.33 \mathrm{GHz}$ Intel Core 2 Duo CPU and 2.00 GB RAM.

Example 3: Consider a transmission line with five segments as follows (for segment numbers see Figure 1.4):

Segment \#1: $5030 \mathrm{ft}, 26$ gauge

Segment \#2: $1320 \mathrm{ft}, 24$ gauge

Segment \#3: 2078 ft, 24 gauge 


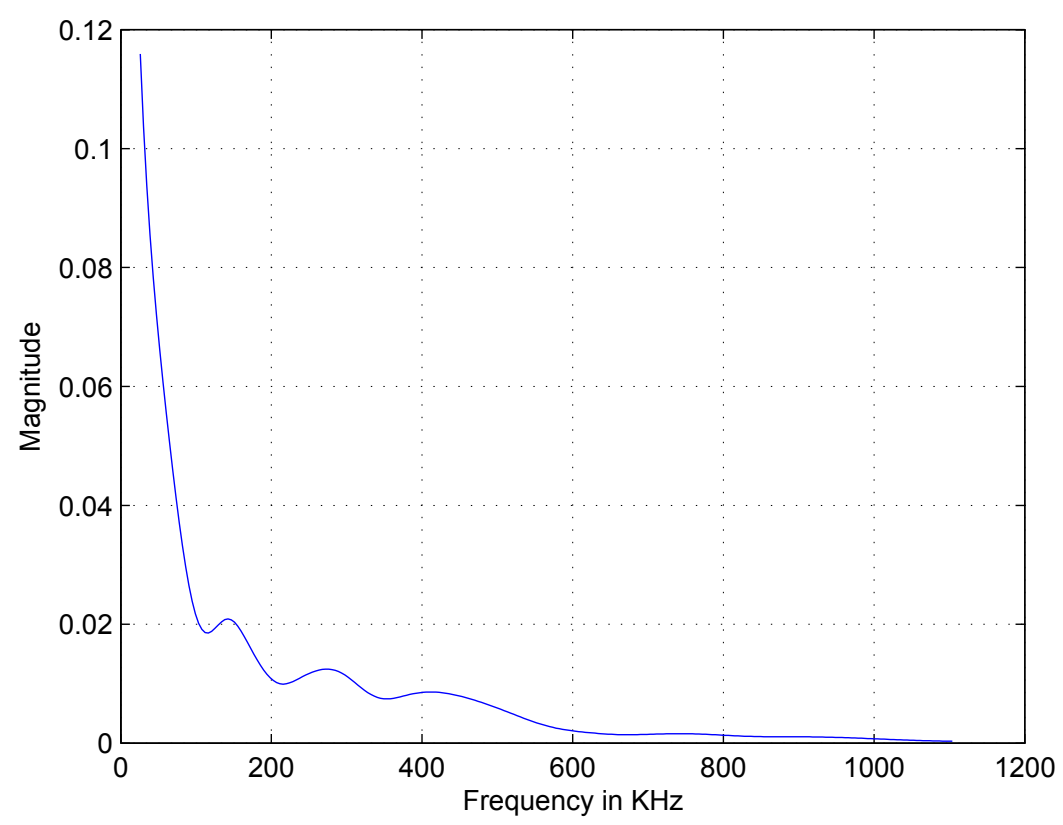

Figure 3.4: Magnitude response for the transmission line of Example 3.

Segment \#4: 709 ft, 24 gauge

Segment \#5: 1013 ft, 22 gauge

The magnitude response of this transmission line is shown in Figure 3.4, and the corresponding information is provided in a bin-by-bin fashion to the initialization and optimization procedures developed in this work. The outcome of the initialization procedure is as follows (for segment numbers see Figure 1.4):

Segment \#1: $4798.6 \mathrm{ft}, 26$ gauge

Segment \#2: $1200 \mathrm{ft}, 26$ gauge

Segment \#3: 2056.5 ft, 24 gauge

Segment \#4: 768 ft, 26 gauge

Segment \#5: $1371 \mathrm{ft}, 22$ gauge 
It can be verified that maximum discrepancy of the initial estimates from the exact values is within $20 \%$. These values are subsequently provided to the main optimization procedure, which arrives at the following values:

Segment \#1: $5031 \mathrm{ft}, 26$ gauge

Segment \#2: $1320 \mathrm{ft}, 24$ gauge

Segment \#3: $2077 \mathrm{ft}, 24$ gauge

Segment \#4: 709 ft, 24 gauge

Segment \#5: 1012 ft, 22 gauge

This demonstrates that the procedure has converged to the exact values for this configuration. The initialization and optimization procedures for this example take $2.27 \mathrm{~min}$ and $0.23 \mathrm{~min}$, respectively, on a personal computer with a $2.33 \mathrm{GHz}$ Intel Core 2 Duo CPU and 2.00 GB RAM.

Example 4: Consider a transmission line with five segments as follows (for segment numbers see Figure 1.4):

Segment \#1: $3000 \mathrm{ft}, 26$ gauge

Segment \#2: 800 ft, 22 gauge

Segment \#3: $1500 \mathrm{ft}, 24$ gauge

Segment \#4: $400 \mathrm{ft}, 22$ gauge

Segment \#5: 2000 ft, 22 gauge 


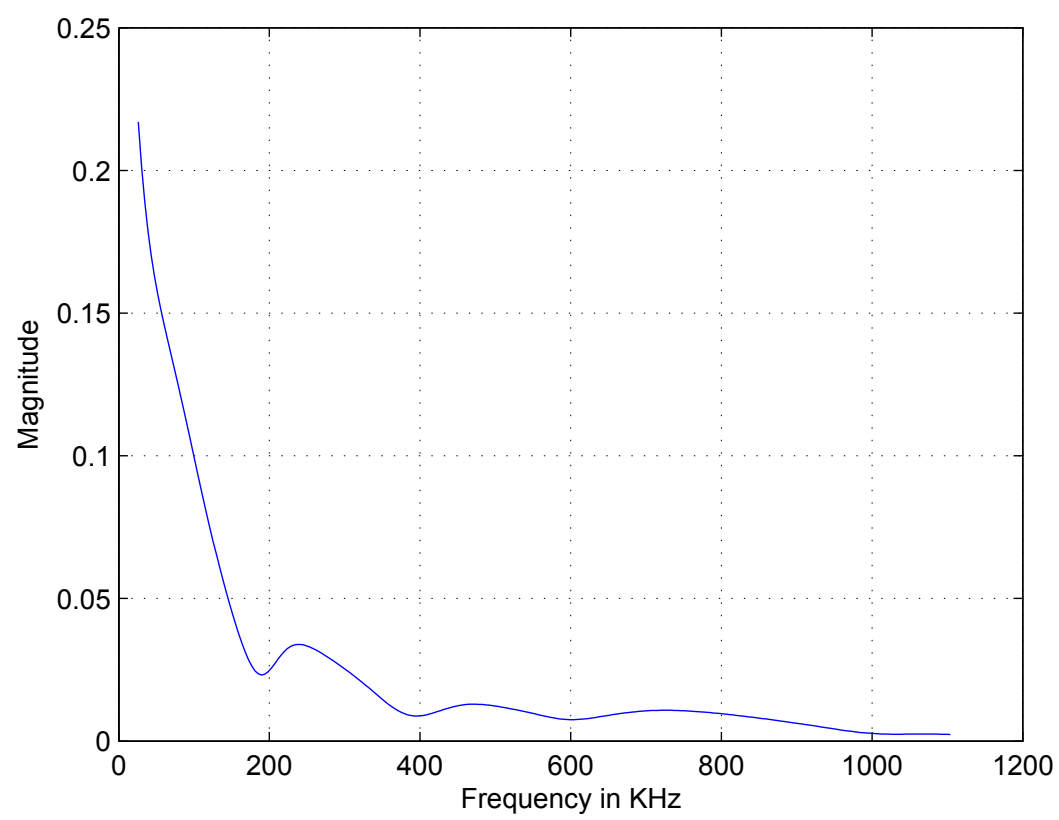

Figure 3.5: Magnitude response for the transmission line of Example 4.

The magnitude response of this transmission line is shown in Figure 3.5, and the corresponding information is provided in a bin-by-bin fashion to the initialization and optimization procedures developed in this work. The outcome of the initialization procedure is as follows (for segment numbers see Figure 1.4):

Segment \#1: $3544.1 \mathrm{ft}, 26$ gauge

Segment \#2: $768 \mathrm{ft}, 22$ gauge

Segment \#3: $1518.9 \mathrm{ft}, 24$ gauge

Segment \#4: 393.2 ft, 22 gauge

Segment \#5: $1012.6 \mathrm{ft}, 22$ gauge

It can be verified that maximum discrepancy of the initial estimates from the exact values 
is within $20 \%$. These values are subsequently provided to the main optimization procedure, which arrives at the following values:

Segment \#1: $3000 \mathrm{ft}, 26$ gauge

Segment \#2: $800 \mathrm{ft}, 22$ gauge

Segment \#3: $1501 \mathrm{ft}, 24$ gauge

Segment \#4: $400 \mathrm{ft}, 22$ gauge

Segment \#5: $2000 \mathrm{ft}, 22$ gauge

This demonstrates that the procedure has converged to the exact values for this configuration. The initialization and optimization procedures for this example take $2.27 \mathrm{~min}$ and $0.23 \mathrm{~min}$, respectively, on a personal computer with a $2.33 \mathrm{GHz}$ Intel Core 2 Duo CPU and 2.00 GB RAM. 


\section{Chapter 4}

\section{Conclusions}

\subsection{Summary}

This thesis presents a technique to identify the configuration of a transmission line in a wired communication network from its frequency response. The proposed technique aims to find the closest match for the given magnitude response, using the integral of squared error as the measure of closeness. The procedure consists of two phases: initialization and optimization. The initialization phase provides a reasonable initial estimate of line parameters for the optimization program which uses direct search method to find the optimal solution. It is assumed that the transmission line consists of not more than two bridged taps, and that the length of bridged taps and main channel are within certain ranges in accordance with the DSL loop standards. 
Chapter 2 provides a technique to estimate the total length of the main line. To this end, the magnitude response of the line is approximated by a polynomial of a sufficiently high degree. A procedure is subsequently used to find an estimate of the line length, mainly from the magnitude response in lower frequencies. While this estimate is not used in the technique presented later in the thesis, it is very useful as it provides an estimate of the distance between the customer and central office, which is very important for the DSL service providers.

The main contribution of the work is provided in Chapter 3, where a technique is presented to identify the configuration of the line (in terms of length and gauge of each segment). An initial estimate of the line configuration is made first, and then an optimization procedure is used to tune the parameters of the model to minimize the difference between the real frequency response of the line and that of the model.

The results of this work can be used as a single-ended testing of the line from the central office to characterize it and determine any sources of possible problems in the signal transmission. Simulations demonstrate the effectiveness of the proposed technique.

\subsection{Future Work}

The proposed algorithm fully characterizes the twisted-pair transmission line with high accuracy from its frequency response. The following issues can be considered for future work: 
i) The overall performance of the procedure depends on the accuracy of the optimal parameters, and how fast they are obtained. This, in turn, depends on the accuracy of the initialization process and how fast it is completed. A more accurate initial estimate would require a longer time, but leads to more accurate parameters (as the output of the optimization procedure) in a shorter period of time. This introduces a trade-off between the accuracy of initialization algorithm and the speed of the optimization procedure, which needs to be addressed by simulations for a more efficient algorithm.

ii) If the lengths of a bridged tap is equal (or very close) to the length of another bridged tap, the initialization algorithm may not distinguish them. A different approach should be used in such cases to estimate the number of bridged taps in the line, before the initialization algorithm is performed. 


\section{Bibliography}

[1] T. Starr, J.M. Cioffi and P.J. Silverman, Understanding Digital Subscriber Line Technology, New York: Prentice Hall, 1999.

[2] S. Galli and K.J. Kerpez, "Single-Ended Loop Make-Up Identification-Part I: Improved Algorithms and Performance Results," IEEE Transactions on Instrumentation and Measurement, vol. 55, no. 2, pp. 528-537, April 2006.

[3] S. Galli and K.J. Kerpez, "Single-Ended Loop Make-Up Identification-Part II: A Method of Analyzing TDR Measurements," IEEE Transactions on Instrumentation and Measurement, vol. 55, no. 2, pp. 538-549, April 2006.

[4] G. Hong, ”Performance of a 51.84-Mb/s VDSL Transceiver Over the Loop with Bridged Taps," IEEE Transactions on Communications, vol. 50, no. 5, May 2002.

[5] J.J. Werner, "The HDSL Environment," IEEE Journal on Selected Areas in Communications, vol. 9, no. 6, August 1991.

[6] P. Boets, T. Bostoen, L.V. Biesen and T. Pollet, "Pre-Processing of Signals for Single-Ended Subscriber Line Testing," IEEE Transactions on Instrumentation and 
Measurement, vol. 55, no. 5, pp. 1509-1518, 2006.

[7] S. Galli and D.L. Waring, "Loop Makeup Identification Via Single Ended Testing: Beyond Mere Loop Qualification,” IEEE Journal on Selected Areas in Communications, vol. 20, no. 5, June 2002.

[8] International Telecommunication Union, Telecommunication G.996.1, Test Procedures for Digital Subscriber Line (DSL) Transceiver, 2001.

[9] S. Galli, "Exact Conditions for the Symmetry of a Loop," IEEE Communications Letters, vol. 4, no. 10, October 2000.

[10] K.J. Kerpez, "The Telcordia DSL Spectral Compatibility Computer website," http://net3.argreenhouse.com, available online.

[11] K. Kerpez and S. Galli, ” Joint Optimization of Single-Carrier and Multi-Carrier DSL Spectra,” IEEE Int. Conf. on Communications, pp. 1347-1351, May 2005.

[12] K.B. Song, S.T. Chung, G. Ginis and J.M. Cioffi, ”Dynamic Spectrum Management for Next-Generation DSL Systems," IEEE Communications Mag., vol. 40, no. 10, pp. 101-109, October 2002.

[13] K. Kerpez, D. Waring, J. Dixon, P. Mandon and S. Galli, ”Advanced DSL Management," IEEE Communications Mag., vol. 41, no. 9, pp. 116-123, September 2003.

[14] W.Y. Chen, DSL-Simulation Techniques and Standards Development for Digital Subscriber Line Systems, Indianapolis, Macmillan Technical Publishing.

[15] I. Kalet and S. Shamai-Shitz, "On the Capacity of a Twisted-Wire Pair: Gaussain Model," IEEE Transactions on Communications, vol. 38, no. 3, pp. 379-383, March 1990. 
[16] Transmission Systems for Communications, Holmdel, NJ: Bell Laboratories, 1982.

[17] J.M. Cioffi, "Very-High-Speed Digital Subscriber Lines-System Requirements," ANSI Contribution T1E1.4/98-172, June 1998.

[18] T. Vermeiren, T. Bostoen, F. Louage, P. Boets and X.O. Chehab, ”Subscriber Loop Topology Classification by Means of Time Domain Reflectrometry," IEEE Int. Conf. on Communications, vol. 3, pp. 1998-2002, May 2003.

[19] P. Boets, T. Bostoen, D. Gardan and L.V. Biesen, "Single Ended Line Testing-A White Box Approach," in Proceedings of 4th IASTED Int. Multi-Conf. Wireless and Optical Communications, pp. 393-398, July 2004.

[20] P.D. McFadden, Interpolation Techniques for the Time Domain Averaging of Vibration Data with Application to Helicopter Gearbox Monitoring, Dept. of Defence, Defence Science and Technology Organisation, Aeronautical Research Laboratories, 1986.

[21] J.C. Lagarias, A.J. Reeds, M.H. Wright and P.E. Wright, ”Convergence Properties of the Nelder Mead Simplex Method in Low Dimensions," SIAM Journal on Optimization, vol. 9, no. 1, pp. 112-147, 1998.

[22] A.P. Gurson, Simplex Search Behavior in Nonlinear Optimization, Bachelors Honors Thesis, Computer Science Department, College of William and Mary, Williamsburg, Virginia, 2000.

[23] T.G. Kolda, R.M. Lewis and V. Torczon, "Optimization by Direct Search: New Perspectives on Some Classical and Modern Methods," SIAM Review, no. 45, pp. 385-482, 2003. 
[24] R.M. Lewis, V. Torczon and M.W. Trosset, "Direct Search Methods: Then and Now, in Numerical Analysis," SIAM Journal on Optimization, vol. 4, pp. 191-207, 2001.

[25] K.I.M. McKinnon, "Convergence of the Nelder Mead Simplex Method to a NonStationary Point,” SIAM Journal on Optimization, vol. 9, pp. 148-158, 1998.

[26] J.A. Nelder and R. Mead, "A Simplex Method for Function Minimization," Computer Journal, no. 7, pp. 308-313, 1965.

[27] J.M. Ortega and W.C. Rheinboldt, Iterative Solution of Nonlinear Equations in Several Variables, Academic Press, New York, 1970.

[28] A. Schrijver, Theory of Linear and Integer Programming, John Wiley and Sons, New York, 1987.

[29] A.L. Garcia, Probability, Statistics, and Random Processes for Electrical Engineering, Prentice Hall, 2008.

[30] A.M. Stuart and A.R. Humphries, Dynamical Systems and Numerical Analysis, Cambridge University Press, Cambridge, UK, 1996.

[31] M.H. Wright, Direct Search Methods: Once Scorned, Now Respectable, AddisonWesley Longman, pp. 191-208, 1996.

[32] V. Torczon, "On the Convergence of Pattern Search Algorithms," SIAM Journal on Optimization, no. 7, pp. 1-25, 1997.

[33] W.H. Press, B.P. Flannery, S.A. Teukolsky and W.T. Vettering, Numerical Recipes in Fortran: the Art of Scientific Computing, Cambridge University Press, Cambridge, UK, 1992. 
[34] C.J. Price, I.D. Coope and D. Byatt, "A Convergent Variant of the Nelder Mead Algorithm," Journal of Optimization Theory and Applications, no. 113, pp. 5-19, 2002.

[35] A.S. Rykov, "Simplex Algorithms for Unconstrained Optimization," Problems of Control and Information Theory, no. 12, pp. 195-208, 1983.

[36] P. Tseng, "Fortified-Descent Simplicial Search Method: A General Approach," SIAM Journal on Optimization, vol. 10, no. 1, pp. 269-288, 1999.

[37] L.J. Nazareth and P. Tseng, "Gilding the Lily: A Variant of the Nelder Mead Algorithm Based on Golden Section Search," Computational Optimization and Applications, vol. 22, pp. 133-144, 2002.

[38] MATLAB User Guide, R2010b Documentation, The Mathworks Inc., Natick, Massachusetts, 2010.

[39] F. Klein, Elementary Mathematics from an Advanced Standpoint: Geometry, Dover Publications: New York, 1939.

[40] L. Han and M. Neumann, "Effect of Dimensionality on the Nelder Mead Simplex Method," Optimization Methods and Software, vol. 21, pp. 1-16, 2006.

[41] A.R. Conn, K. Scheinberg and L.N. Vicente, "Introduction to Derivative-Free Optimization," SIAM Journal on Optimization, no. 53, pp. 395-396, 2009.

[42] I.D. Coope and C.J. Price, "On the Convergence of Grid-Based Methods for Unconstrained Optimization," SIAM Journal on Optimization, vol. 11, pp. 859-869, 2001.

[43] C. Audet and J.E. Dennis, "Analysis of Generalized Pattern Searches," SIAM Journal on Optimization, vol. 13, pp. 889-903, 2003. 
[44] C. Audet and J.E. Dennis, "Mesh Adaptive Direct Search Algorithms for Constrained Optimization,” SIAM Journal on Optimization, vol. 17, pp. 188-217, 2006.

[45] Characterization of Subscriber Loops for Voice and ISDN Services (1983 subscriber loop survey results), Bellcore, Science and Technology Series, ST-TSY-000 041, 1987.

[46] Transmission and Multiplexing (TM); Access Transmission Systems on Metallic Access Cables; Very High Speed Digital Subscriber Line (VDSL); Part 2: Transceiver Specification, ETSI TS 101 270-2, 2001.

[47] G. Ginis and J.M. Cioffi, ”Vectored Transmission for Digital Subscriber Line Systems," IEEE Journal on Selected Areas in Communications, vol. 20, no. 5, pp. 10851104, June 2002.

[48] A. Wang, J.J. Werner and S. Kallel, Effect of Bridged Taps on Channel Capacity at VDSL Frequencies, Bell Laboratories, Holmdel, USA.

[49] B. Porat and D. Schmucking, "The Achievable Capacity of Fust-Generation FDD Symmetric VDSL Under the FSAN Noise Model," ETSI Contribution, TD-30, January 1998.

[50] T. Bostoen, P. Boets, M. Zekri, L.V. Biesen, T. Pollet and D. Rabijns, ”Estimation of the Transfer Function of a Subscriber Loop by Means of a One-Port Scattering Parameter Measurement at the Central Office," IEEE Journal on Selected Areas in Communications, vol. 20, no. 5, pp. 936-948, June 2002.

[51] W.D. Block, R. Pintelon and Y. Rolain, "Identification of Transmission Lines: From Time Domain Measurements to Frequency Domain Models," IEEE Instrumentation and Measurement Technology Conference, May 2002. 


\section{Appendix A}

\section{Appendix}

\section{A.1 MATLAB Code to Determine the Length of Bridged}

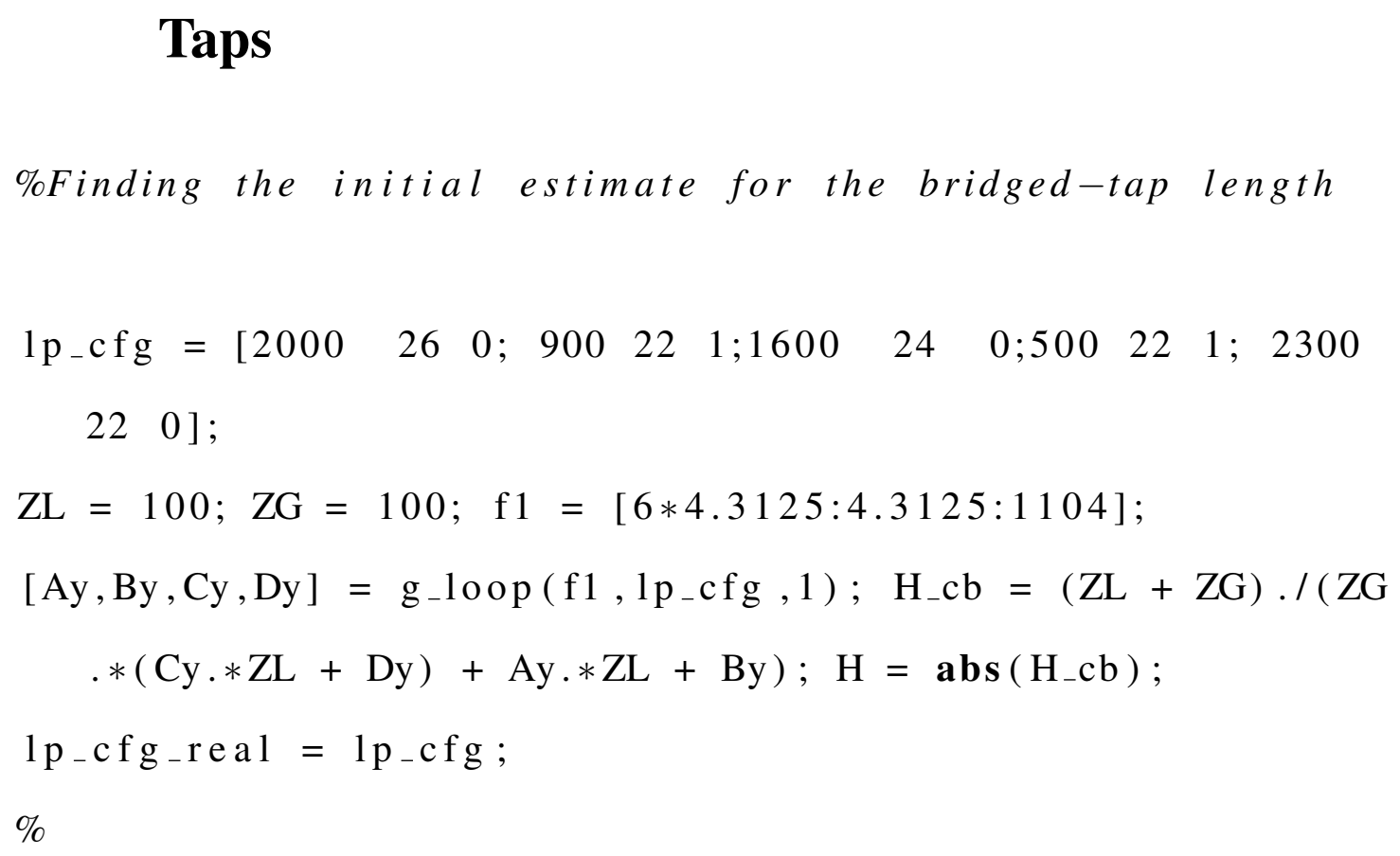




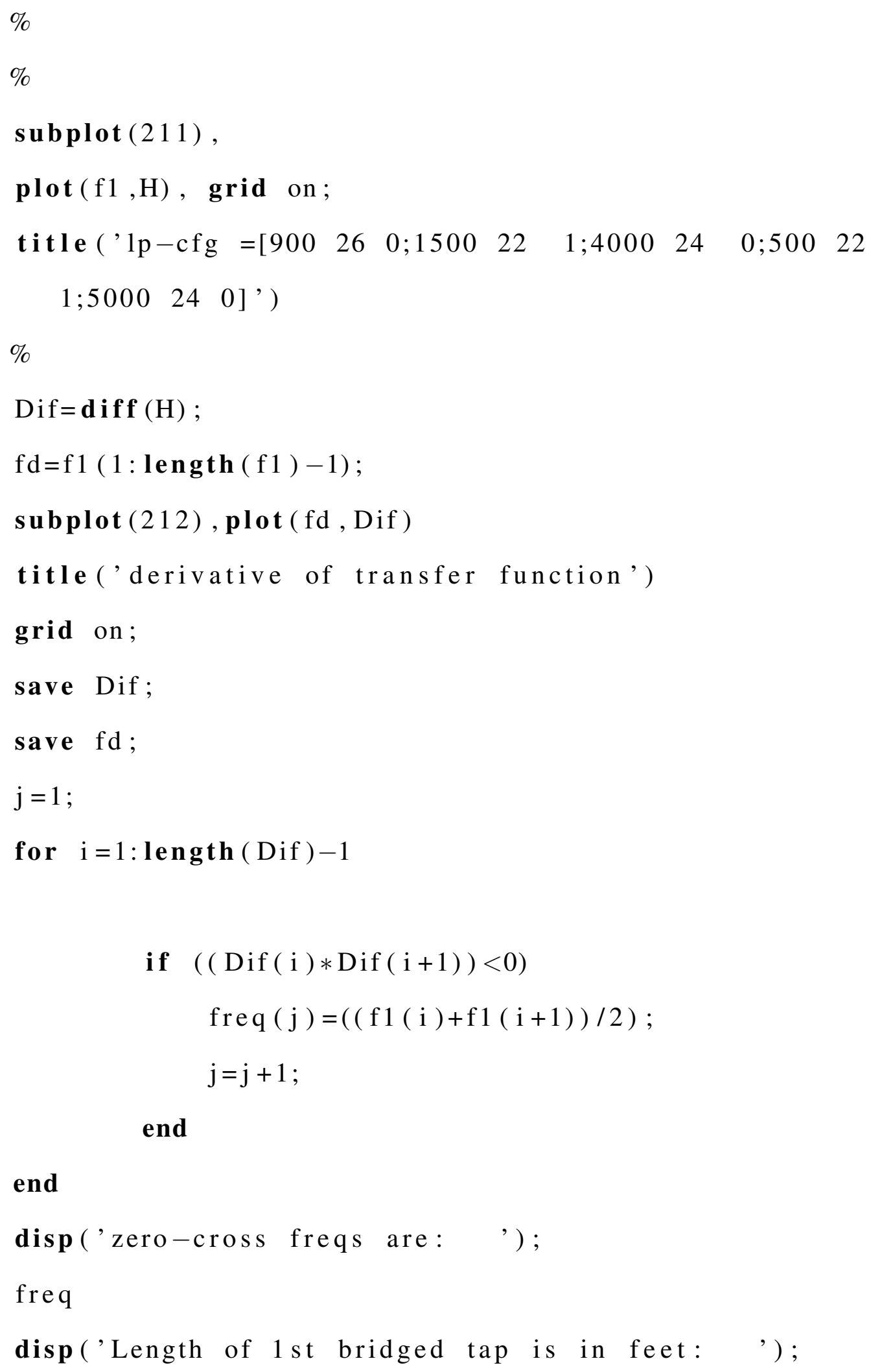


yy $1=150000 . /$ freq $(1)$

disp('Length of 2 nd bridged tap is in feet: ');

yy $2=150000 . /$ freq ( 3$)$

\section{A.2 MATLAB Code to Determine the Total Length of}

\section{Line}

\%Equation generated by the fitting curve.

$\%$ function $y=C F(f)$

$\% \quad p l=-5.9324 e-008 ;$

$\% \quad p 2=2.0479 e-005$;

$\% \quad p 3=-0.0043668$;

$\% \quad p 4=0.64293$;

$\%$

$\% y=p 1 * f^{\wedge} 3+p 2 * f^{\wedge} 2+p 3 * f+p 4 ;$

$\% \%$ Equation generated by theory.

$\%$ function $h=L L(f, x)$

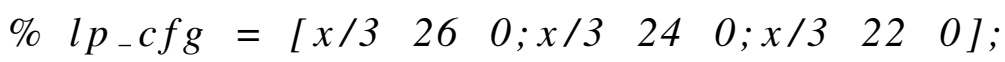

$\% Z L=100 ; Z G=100$;

$\%[A y, B y, C y, D y]=g_{-} l o o p\left(f, l p_{-} c f g, l\right) ; H_{-} c b=(Z L+Z G) . /(Z G$

$$
\cdot *(C y \cdot * Z L+D y)+A y \cdot * Z L+B y) ;
$$

$\% h=a b s\left(H_{-} c b\right)$;

$\% \%$ Function generation for direct search.

$\%$ function $F=L L F(y)$ 


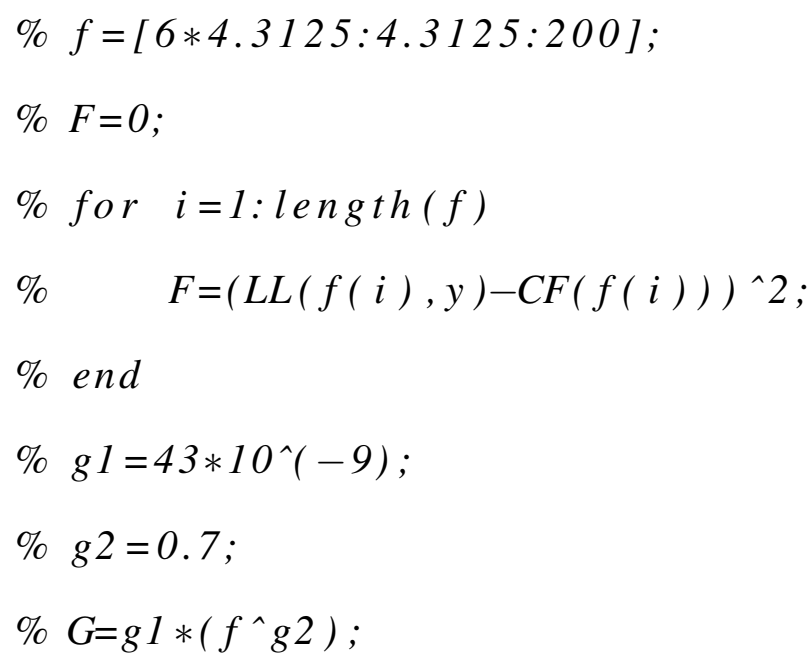


$\% \quad Z l=Z$;

$\%$ phi $1=[\cosh ($ gamma $* d 1) \quad Z * \sinh ($ gamma $* d 1) ;(1 / Z) * \sinh ($ gamma $* d 1$ ) $\cosh ($ gamma $* d 1)]$;

$\%$ phi2 $=\left[\begin{array}{ll}1 & 0 ;(1 / Z) * \tanh (\text { gamma } * d 2) \quad 1\end{array}\right]$

$\%$ phi3 $=[\cosh ($ gamma $* d 3) \quad Z * \sinh ($ gamma $* d 3) ;(1 / Z) * \sinh ($ gamma $* d 3$

) $\cosh (\operatorname{gamma} * d 3)]$;

$\%$ phi4 $=\left[\begin{array}{ll}1 & 0 ;(1 / Z) * \tanh (\operatorname{gamma} * d 4) \quad 1\end{array}\right]$;

$\%$ phi5 $=[\cosh ($ gamma $* d 5) \quad Z * \sinh ($ gamma $* d 5) ;(1 / Z) * \sinh ($ gamma $* d 5$

) $\cosh (\operatorname{gamma} * d 5)]$;

$\% \quad p h i=p h i 1 *$ phi $2 *$ phi $3 *$ phi $4 *$ phi 5 ;

$\% \% p h i=p h i 1 *$ phi3 $*$ phi5;

$\% H=a b s(Z l /((p h i(1,1) * Z l)+p h i(1,2)+p h i(2,1) * Z l * Z s+p h i(2,2) *$ $Z s)$ )

\section{A.3 MATLAB Code to Deal with the Variable Nature of $R L C G$ Values with Frequency}

clc

clear all

\%per unit value of $26 A W G$

\%syms f

$\mathrm{f}=[6 * 4.3125 * 1000: 4.3125: 1104 * 1000]$;

r1 $=286.17578$;

$\mathrm{r} 2=\mathrm{inf}$; 


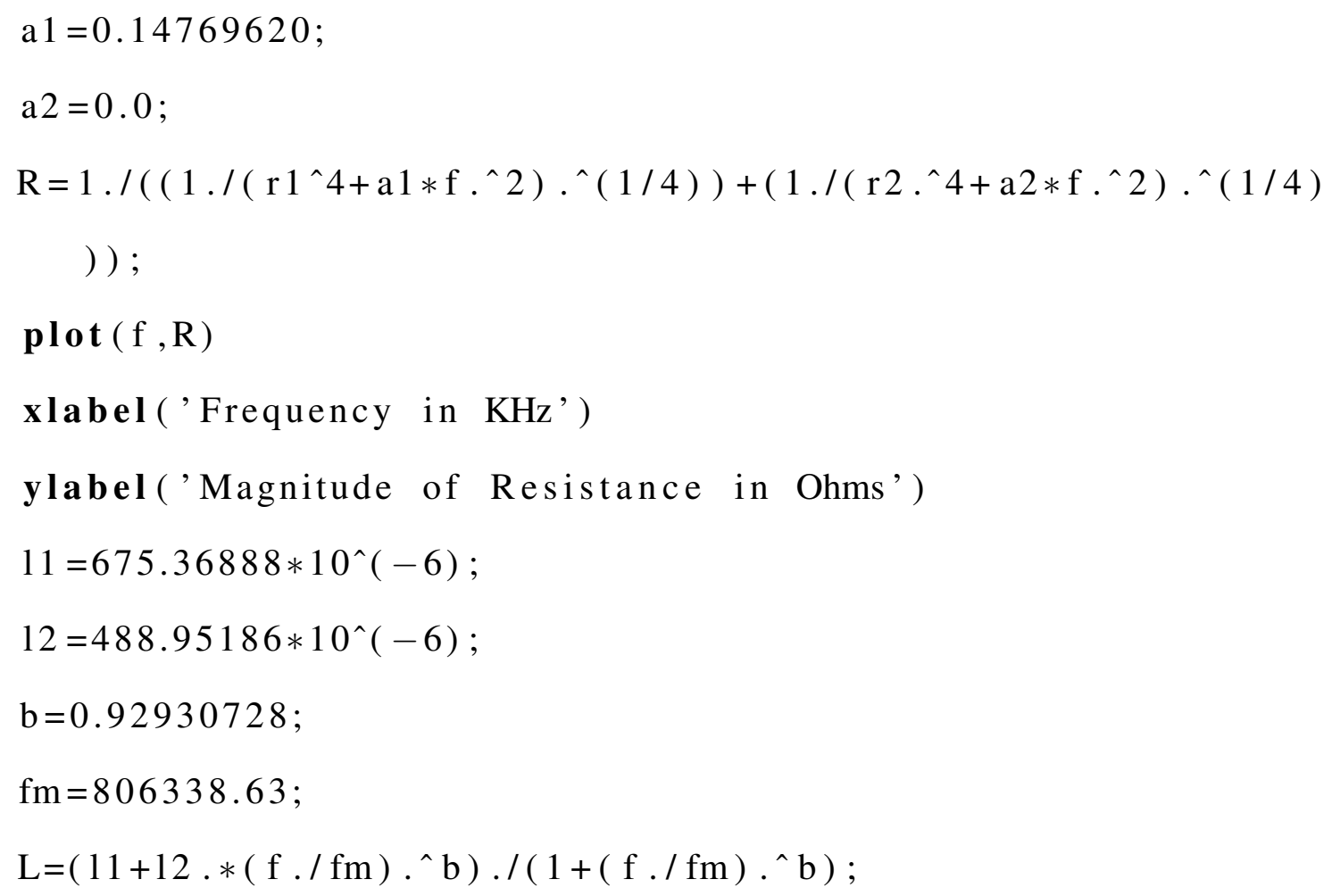

\%per unit value of $24 A W G$

$\% R=175$

$\% L=616 e-6$;

$\% C=50 e-9$; 
$\% G=0.0$;

$\%$

$\% d l=3$;

$\% d 2=0.1$;

$\% d 3=1.0$;

$\% d 4=0.1$;

$\% d 5=1$;

$\%$

$\%$

$\%$ gamma $=\operatorname{sqrt}((R+i * 6.283185 * f * L) *(G+i * 6.283185 * f * C))$;

$\% Z=\operatorname{sqrt}((R+i * 6.283185 * f * L) /(G+i * 6.283185 * f * C))$;

$\% Z s=Z$;

$\% \quad Z l=Z$;

$\%$ phi $1=[\cosh ($ gamma $* d 1) \quad Z * \sinh ($ gamma $* d 1) ;(1 / Z) * \sinh ($ gamma $* d 1$ ) $\cosh (\operatorname{gamma} * d 1)]$;

$\%$ phi2 $=\left[\begin{array}{ll}1 & 0 ;(1 / Z) * \tanh (\operatorname{gamma} * d 2) \quad 1\end{array}\right] ;$

$\%$ phi3 $=[\cosh ($ gamma $* d 3) \quad Z * \sinh ($ gamma $* d 3) ;(1 / Z) * \sinh (\operatorname{gamma} * d 3$ ) $\cosh (\operatorname{gamma} * d 3)]$;

$\%$ phi4 $=\left[\begin{array}{ll}1 & 0 ;(1 / Z) * \tanh (\operatorname{gamma} * d 4) \quad 1\end{array}\right]$

$\%$ phi5 $=[\cosh ($ gamma $* d 5) \quad Z * \sinh ($ gamma $* d 5) ;(1 / Z) * \sinh ($ gamma $* d 5$ ) $\cosh (\operatorname{gamma} * d 5)]$;

$\% \quad p h i=p h i 1 * p h i 2 *$ phi $3 *$ phi $4 *$ phi 5 ;

$\% \%$ phi=phil $*$ phi3 $*$ phi5;

$\% H=a b s(Z l /((p h i(1,1) * Z l)+p h i(1,2)+p h i(2,1) * Z l * Z s+p h i(2,2) *$ $Z s)$ ) 


\section{Appendix B}

\section{Appendix}

\section{B.1 Modified Section of MATLAB Code for the Initial- ization Phase}

\%Modification subcode in the initialization phase.

$\%$ Using a portion of frequency points which is more informative

$\mathrm{ff}=1000 * \mathrm{f} 1(1: 1: \text { round }(\text { length }(\mathrm{f} 1) / 2))^{\prime} ; \%$ This change of scale is necessary because g-loop uses frequency in $\mathrm{KHz}$.

$\mathrm{f} 1=\mathrm{f} 1(1: 1: \text { round }(\text { length }(\mathrm{f} 1) / 2))^{\prime} ;$

$\mathrm{H}=\mathrm{H}(1: 1:$ round $($ length $(\mathrm{H}) / 2))$;

\%size $(H)$

$\mathrm{f}_{-}$length $=$length $(\mathrm{ff}) ;$ 
$\%$ load the files for g_loop corresponding to different gauges

fid=fopen (['rlgc $\backslash$, '22, ' pic70.txt'], 'r' );

$[\mathrm{y} 1, \mathrm{cc}]=\mathbf{f s c a n f}(\mathrm{fid}, \% \mathrm{f},,[5,37])$;

fclose ( f id ) ;

$\mathrm{fid}=$ fopen $\left(\left[{ }^{\prime} \mathrm{rlgc} \backslash,{ }^{\prime}, 24, \operatorname{pic} 70 . \mathrm{txt}{ }^{\prime}\right],,^{\prime} \mathrm{r}\right.$ ') ;

$[y 2, \mathrm{cc}]=\mathbf{f s c a n f}(\mathrm{fid}, ' \%$ f', $[5,37])$;

f close ( fid ) ;

$\mathrm{fid}=$ fopen ( [ 'rlgc $\backslash$ ' '26,' 'pic70.txt'], 'r' );

$[y 3, c c]=$ fscanf ( fid , '\%f', [ 5,37]);

fclose ( fid ) ;

\% Set the error index to a big initial value

Error $=1 . \mathrm{e} 10$

$\operatorname{config}=[]$

$\mathrm{kk}=1$;

for $b_{-} g=22: 2: 26$,

$\%$ Load prestored values for different lengths and

gauges corresponding to different bridged-tap gauges

if $\mathrm{b}_{-} \mathrm{g}==22$,

load lengthSimulationData_22

else if $b_{-} g==24$,

load lengthSimulationData_24

else

load lengthSimulationData_26 
end

$\%$ Choosing the lines corresponding to the above bridged -tap length (in both locations)

\%yO $=\min (\operatorname{abs}(Y(1: 8,2)-y y)) ;$

$\mathrm{i} 1=\operatorname{find}(\operatorname{abs}(\mathrm{Y}(:, 1)-\mathrm{yy} 1)==\min (\operatorname{abs}(\mathrm{Y}(1: 8,2)-\mathrm{yy} 1))$

$\& \operatorname{abs}(Y(:, 2)-$ yy2 $)==\min (\operatorname{abs}(Y(1: 8,2)-y y 2))) ;$

$\mathrm{Y}=\mathrm{Y}(\mathrm{i} 1,:)$;

H0_22 = H0_22 (:, i 1$)$;

H0_24 = H0_24 ( : , i 1$)$;

H0_26 = H0_26 (:, i 1$)$;

\% Main line segment gauges

$\mathrm{g} 1=\left[\begin{array}{lll}22 & 24 & 26\end{array}\right]$;

$\mathrm{g} 2=\left[\begin{array}{lll}22 & 24 & 26\end{array}\right]$;

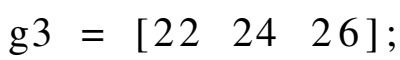

g4 = [llll 224 26];

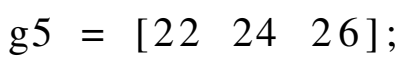

$\%$ Bridged-tap gauges

$\mathrm{G}_{-} \mathrm{y}=\left[\begin{array}{ll}\mathrm{b}_{-} \mathrm{g} & \mathrm{b}_{-} \mathrm{g}\end{array}\right]$;

\% Finding the initial estimate for the line length (one estimate for each combination of bridged-tap lengths)

h0_22 $=\min \left(\operatorname{abs}\left(\mathrm{H}_{0} \_22-\mathrm{H}(1)\right)\right)$;

for $\mathrm{i}=1:$ length $\left(\mathrm{h} 0_{-} 22\right)$, 


$$
\mathrm{d} 1(\mathrm{i})=\mathrm{d}\left(\text { find }\left(\operatorname{abs}\left(\mathrm{H}_{0} \_22(:, \mathrm{i})-\mathrm{H}(1)\right)==\mathrm{h} 0 \_22(\mathrm{i})\right)\right)
$$

end

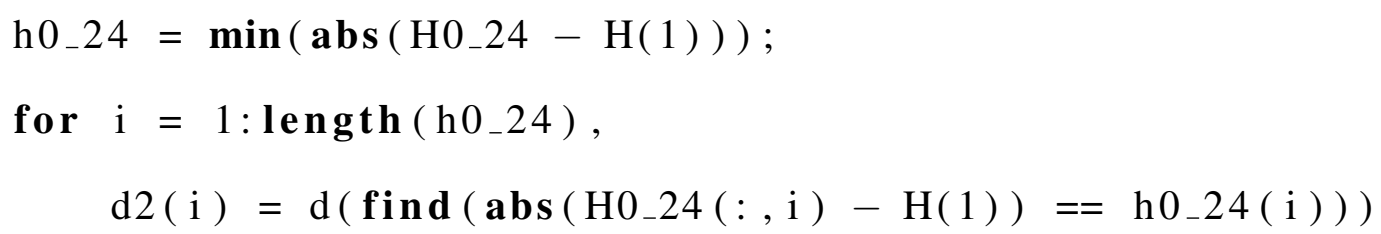

